\title{
UNITED AIRCRAFT CORPORATION
}

RESEARCH IAABORATORIES

East Hartford, Connecticut

UARL Report M921514-18

Semiannual Report

Under Contract AT(11-1)-2277

$$
\text { July 31, } 1972
$$

through

January 31, 1973

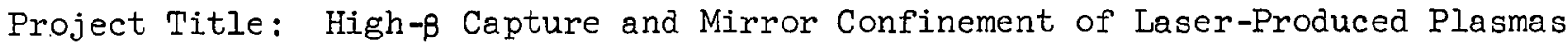
Name of Contractor: United Aircraft Corporation Research Laboratories.

\section{Reported By:}

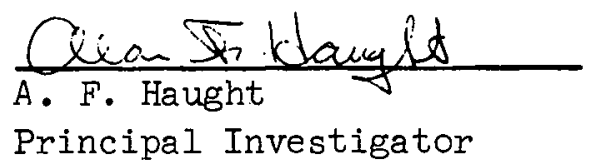

This report was prepared as an account of work The report was prepared as an sponsored by the United States Gover Alomic Energy the United States nor the United States Atomic Energy Commission, nor any of their employees, nor any of

their contractors, subcontractors, or their employees, makes any warranty, express or implied, or assumes any makes anility or responsibility for the accuracy, comlegal lisbilit or pleteness or usefuln disclosed, or represents that its use product or process disclosed, orned rights.
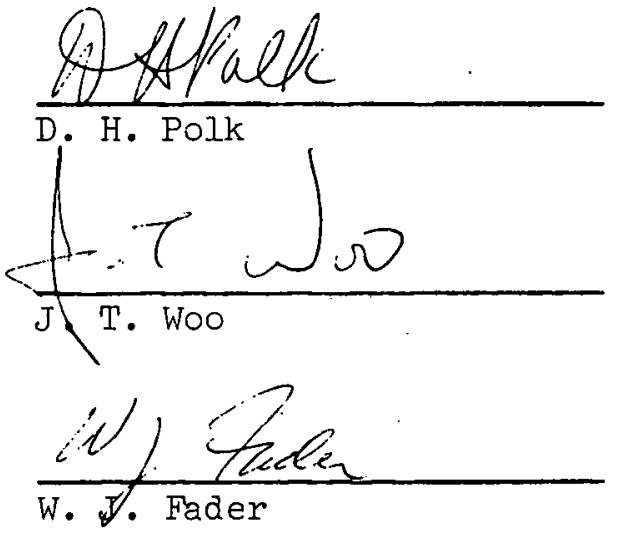



R. G. Tomlinson

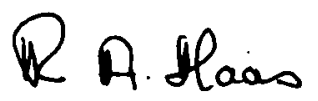

R. A. Haas

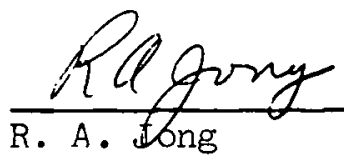




\section{DISCLAIMER}

This report was prepared as an account of work sponsored by an agency of the United States Government. Neither the United States Government nor any agency Thereof, nor any of their employees, makes any warranty, express or implied, or assumes any legal liability or responsibility for the accuracy, completeness, or usefulness of any information, apparatus, product, or process disclosed, or represents that its use would not infringe privately owned rights. Reference herein to any specific commercial product, process, or service by trade name, trademark, manufacturer, or otherwise does not necessarily constitute or imply its endorsement, recommendation, or favoring by the United States Government or any agency thereof. The views and opinions of authors expressed herein do not necessarily state or reflect those of the United States Government or any agency thereof. 


\section{DISCLAIMER}

Portions of this document may be illegible in electronic image products. Images are produced from the best available original document. 
Semiannual Report Under Contract AT(11-1)-2277

For the period August 1, 1972 through January 31, 1973

\section{High- $\beta$ Capture and Mirror Confinement}

of Laser-Produced Plasmas

TABLE OF CONTENTS

I. Summary ........................

$\underline{\text { Page }}$

II. Theoretical Studies . . . . . . . . . . . . . . . . . . . .

A. Target Plasma Calculations. . . . . . . . . . . 2

1. Neutral Beam Injection .............. 2

a. GAIN Code Modifications . . . . . . . . . . 2

i. Ionization and Charge Exchange with the

Neutral Beam ............... 3

ii. Charge Exchange with the Neutral Background. . 5

b. Target Plasma Time History .......... 6

2. Beam and Vacuum Requirements ........... 8

B. Mirror Plasma Confinement ............. 10

1. Mirror-Confined Plasma Decay ........... 10

2. Length and Radial Size Stability Criteria . . . . . 12

III. Experimental Program. . . . . . . . . . . . . 15

A. Diagnostic Development. . . . . . . . . . . 15

1. Laser Interferometer Diagnostics. . . . . . . 15

2. R.F. Emission Diagnostics . . . . . . . . . 16

B. Experimental Progress . . . . . . . . . . . 17

1. System Operation ................ 17

2. Large Particle Suspension ............ 18

3. Plasma Capture and Confinement. . . . . . . . . . 19 
TABLE OF CONTENTS (cont'd)

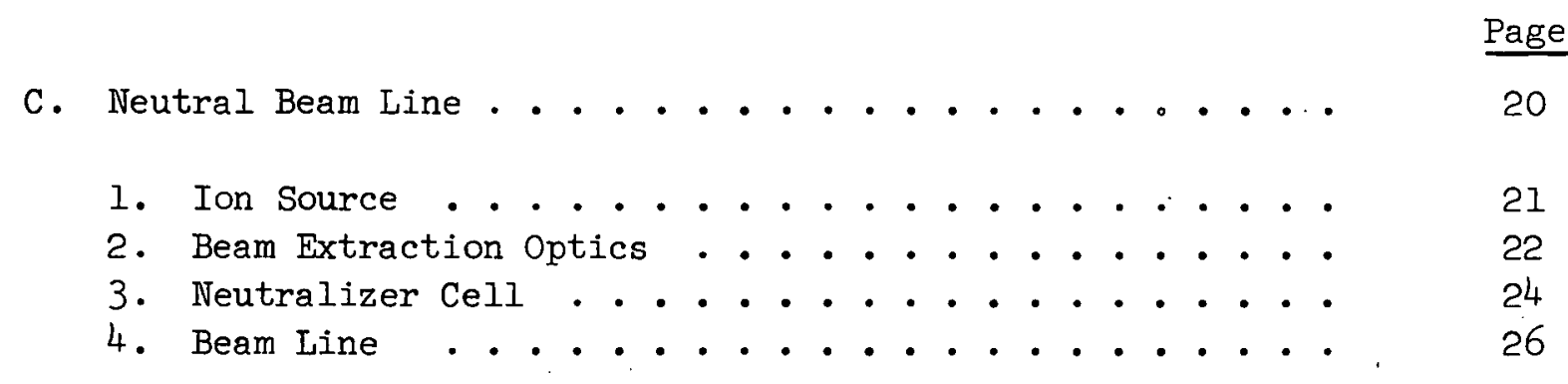

IV. Future Program ....................... 28

REFERENCES . . . . . . . . . . . . . . . . . . . . . . . 30

LIST OF FIGURES . . . . . . . . . . . . . . . . . . . . . . . . 32 
UARL Report M921514-18

\author{
Semiannual Report Under Contract AT(II-I)-2277 \\ For the period August 1, 1972 through January 31, 1973 \\ High- $\beta$ Capture and Mirror Confinement \\ of Laser-Produced Plasmas
}

I. SUMMARY

In the UARL Laser Initiated Target Experiment (LITE), magnetically confined, moderate temperature, dense plasmas produced by laser beam irradiation of a small solid particle in vacuum within a minimum-B mirror field are being utilized as the target for a high energy neutral injection beam to examine high- $\beta$ mirror plasma confinement and the feasibility of neutral injection at high density to produce a hot, dense, steady state, stable, mirror confined plasma. Laser heating of a solid LiH particle electrodynamically suspended in vacuum within the mirror magnetic confinement field initially produces a high density, moderate temperature plasma. Upon expansion, the plasma transverse motion is stopped by the magnetic field, and, by virtue of the high plasma conductivity, the plasma is captured at $\beta=1$. As the magnetic field subsequently diffuses into the plasma, it undergoes a transition to $\beta \leq 1$ mirror confinement. Previous investigations in this program have shown that the resulting plasma can be stably confined in a minimum-B mirror magnetic field, decaying at a rate consistent with Coulomb collisional scattering into the magnetic field mirror loss cones. The investigations currently in progress involve examination of the transition from initial capture with the magnetic field excluded from the plasma volume to $\beta<1$ mirror confinement and extension of the earlier confinement results to higher plasma temperatures in the larger volume, higher field, baseball seam, minimum-B magnet coil constructed for the target plasma experiments. In addition, studies are being made of the filling of the mirror confinement region with increased quantities of plasma produced from bigger suspended particles to determine the scaling to larger magnetic field geometries. For the future target plasma experiments, a LiH particle will again be used for the initial laser plasma production. As demonstrated in the earlier studies, the higher charge state of the lithium ions leads to their rapid decay from the confinement region. Upon injection of the energetic neutral hydrogen beam, calculations show that charge exchange collisions replace the moderate energy hydrogen ions of the confined laser produced plasma with the high energy hydrogen atoms from the beam. The charge exchange interaction will result in heating the contained plasma to a temperature determined by the injected beam energy, reducing the Coublomb loss rate. As the plasma decays, Fokker-Planck calculations show that for a sufficiently high beam current and target density a balance is established between the rate of particle input to the plasma by ionization of the injected beam on the target and the losses due to Coulomb scattering, charge exchange with the cold neutral background, and nonadiabaticity to form a steady state mirror confined plasma. A most attractive feature of the LITE program is that it permits addressing the target plasma questions crucial to the future of open confinement systems with experimental requirements that lie within the current stateof-the-art. 


\section{THEORETICAL STUDIES}

\section{A. Target Plasma Calculations}

\section{Neutral Beam Injection}

The Fokker-Planck calculations of the decay of a mirror confined hydrogen plasma reported previously (Ref. 1) have been extended to include the effects of charge exchange and ionization and employed to follow the time evolution of a dense mirrorconfined laser-produced plasma used as the target for an energetic neutral injection beam. These calculations use as their basis the GAIN code (Refs. 2 and 3) developed by Killeen and co-workers at the Lawrence Livermore Laboratory as adapted for the decay studies and with the additional features described in detail in Ref. 1. For the neutral injection investigations the code has been further modified to incorporate the effects of ionization of the energetic beam neutrals, charge exchange of the beam neutrals with the plasma ions, and plasma losses resulting from charge exchange with the vacuum system neutral background.

\section{a. GAIN Code Modifications}

In the GAIN code the space-independent electron and ion velocity space distribution functions, $f(v, t)$ and $g(v, t)$, are calculated as the solution of a pair of coupled Fokker-Planck equations for the lowest eigen-mode of the angular velocity distribution in a magnetic square well. The physical parameters of plasma density and electron and ion temperatures are computed in turn as appropriate moments of the resulting time dependent distribution functions. The coupled Fokker-Planck equations as modified for the neutral injection calculations may be written in the form:

$$
\begin{aligned}
& \frac{d f}{d t}=\left(\frac{\partial f}{\partial t}\right)_{F C}+\left(\frac{\partial f}{\partial t}\right)_{I O N}-\left(\frac{\partial f}{\partial t}\right)_{L C} \\
& \frac{d g}{d t}=\left(\frac{\partial g}{\partial t}\right)_{F C}+\left(\frac{\partial g}{\partial t}\right)_{I O N}-\left(\frac{\partial g}{\partial t}\right)_{L C}-\left(\frac{\partial g}{\partial t}\right)_{C \times O}+\left(\frac{\partial g}{\partial t}\right)_{C \times 1}-\left(\frac{\partial g}{\partial t}\right)_{C \times 2}
\end{aligned}
$$

The first two terms, (. ) FC -()$_{I C}$, in both Eqs. 1 and 2 represent the FokkerPlanck operator for collisional relaxation and the correction to it accounting for scattering into the loss cones. These terms in Eqs. 1 and 2 are identical with the right hand sides of Eqs. 7 and 8 of Ref. I used for the earlier plasma decay calculations. For the neutral beam injection investigations, the equation for the electron distribution function includes an additional term representing the source of electrons resulting from collisional ionization of the neutral beam. The ionization electrons are assumed to have initially the speed distribution of the neutral beam atoms and their subsequent redistribution in velocity space is accounted for by the FokkerPlanck term, $\left(\frac{\partial f}{\partial t}\right)_{\mathrm{FC}} \cdot$ Collisions with other electrons tend to relax the electron 
distribution to a Maxwellian corresponding to the mean electron energy, while collisions with ions tend to equalize the mean energies of the electrons and ions. The equation for the ion distribution function of Ref. 1 has, similarly, been augmented by an ionization source term and, in addition, by three terms representing the effects of charge exchange. The first of these $-\left(\frac{\partial g}{\partial t}\right)$ cxo' accounts for charge exchange with the neutral background. This process replaces plasma ions by cold ions of the neutral background gas, which are immediately lost from the plasma by scattering into the loss cones. The remaining two terms, $\left(\frac{\partial g}{\partial t}\right)$ cxf $-\left(\frac{\partial g}{\partial t}\right)$ cxe' describe charge exchange between plasma ions and the atoms of the neutral beam. These added terms in the electron and ion distribution function equations are discussed in detail in the following two sections.

\section{i. Ionization and Charge Exchange with the Neutral Beam}

Ionization of the incident neutral beam particles by collisions with the plasma ions and electrons constitutes the source of additional plasma particles which acts to offset the plasma losses. The general form for the rate of change of a particle distribution function $f_{I}(v, t)$ as the result of a reaction with cross-section $\sigma$ between neutral beam particles with speed distribution $f_{B}(v)$ and plasma particles with distribution $f_{2}(v, t)$ is given by

$$
\frac{\partial f_{1}}{\partial t}(v, t)=\int f_{B}(v) f_{2}(u, t)|v-u| \sigma(|v-u|) d^{3} u
$$

Here $\mathrm{v}$ and $\mathrm{u}$ are, respectively, the beam and plasma particle velocities, and the cross-section $\sigma$ is assumed to be a function only of the relative speed of the interacting particles. The ionization source term for electrons and for ions includes two such integrals, one representing ionization of the beam neutrals by electron collisions, the other by ion collisions. Consistent with other approximations involved in this calculation, the electron ionization cross-section $\sigma$ is assumed to be a constant, $10^{-16} \mathrm{~cm}^{2}$, for electron speeds larger than a cut-off, $v$, and zero for smaller speeds. The cut-off is the speed of an electron with energy equal to the ionization potential of the hydrogen beam atom. Similarly, the ionization cross-section for ion collisions with beam atoms is assumed to be $10^{-16} \mathrm{~cm}^{2}$ for ion speeds above the corresponding ion cut-off speed, $v_{c i}$, and zero below. The electron source term obtained after carrying out the integration over all directions of $u$ has the form,

$$
\begin{aligned}
& \left(\frac{\partial f}{\partial t}\right)_{1 O N}=4 \pi \sigma_{e} f_{B}(v)\left\{v \int_{v_{C e}}^{v} f(u)\left(1+\frac{1}{3}\left(\frac{u}{v}\right)^{2}\right) u^{2} d u+\int_{v}^{\infty} f(u)\left(1+\frac{1}{3}\left(\frac{v}{u}\right)^{2}\right) u^{3} d u\right\} \\
& \quad+4 \pi \sigma_{i} f_{B}(v)\left\{v \int_{v_{C i}}^{v} g(u)\left(1+\frac{1}{3}\left(\frac{u}{v}\right)^{2}\right) u^{2} d u+\int_{v}^{\infty} g(u)\left(1+\frac{1}{3}\left(\frac{v}{u}\right)^{2}\right) u^{3} d u\right\}
\end{aligned}
$$


Since ions and electrons produced by ionization of the neutral beam are added to the plasma with the same speed distribution, $f_{B}(v)$, the ion source term is identical with that for the electrons, and

$$
\left(\frac{\partial g}{\partial t}\right)_{I O N}=\left(\frac{\partial f}{\partial t}\right)_{1 O N}
$$

A charge exchange collision between a plasma ion and the energetic atom of the neutral beam results in the addition of an energetic ion to the plasma and the loss as a neutral of an ion from the plasma ion distribution. This reaction rate is also represented in general form by $\mathrm{Eq} .3$ and constitutes a redistribution of the plasma energy with particle number conservation. In evaluating the beam-plasma charge exchange terms, the $\mathrm{H}-\mathrm{H}+$ charge exchange cross-section was approximated by the expression

$$
\sigma_{c x}=\left(7.6 \times 10^{-8}-1.06 \times 10^{-8} \log _{10} E\right)^{2} \quad \mathrm{~cm}^{2}
$$

suggested by Fite, et. al. (Ref. 4) as the best fit to the experimental data. To simplify the integration, the relative speed of reacting particles was approximated by the beam particle speed. The rate of gain of energetic ions by charge exchange with the neutral beam is then given by

$$
\left(\frac{\partial g}{\partial t}\right)_{c x \mid}=4 \pi f_{B}(v)(a-b \ln v)^{2} v \int_{0}^{\infty} g(u) u^{2} d u
$$

and the rate of loss of plasma ions by this process is

$$
\left(\frac{\partial g}{\partial t}\right)_{c x 2}=4 \pi g(v) \int_{0}^{\infty} f_{B}(u)(a-b \ln u)^{2} u^{3} d u
$$

The values of the constants $a$ and $b$ correspond to the numerical coefficients in the charge exchange cross-section $\sigma_{\mathrm{cx}}$.

The velocity distribution of the neutral beam, $f_{B}(v)$, was taken for the calculations as a Gaussian function, centered at the speed $v_{B}$ corresponding to the beam energy $E_{B}$. The beam energy spread, full width at half-intensity, was chosen as twenty percent of $E_{B}$, selected as typical of the energy spread achievable with neutral beams, and the beam distribution function was normalized to the neutral particle current $I$, by the relation

$$
\frac{I L}{v}=4 \pi \int_{0}^{\infty} f_{B}(v) v^{2} d v
$$

in which $L$ is the mean chord length of the beam path and $V$ is the plasma volume. 


\section{ii. Charge Exchange with the Neutral Background}

Charge exchange collisions with the neutral background atoms of the vacuum system result in the replacement of a plasma ion by a. low energy impurity ion, which is immediately lost by scattering into the loss cones. Two kinds of neutrals are involved: cold neutrals of the residual gas of the vacuum system with energies of about $.025 \mathrm{eV}$, and Franck-Condon neutrals with energies of about $10 \mathrm{eV}$. The cold background neutrals have mean free paths in the high density plasma much smaller than the plasma dimensions, and they are ionized or charge-exchanged near the surface of the plasma. Although not important from an energy loss or particle gain standpoint, ionization can result in an excited, repulsive state molecule. The dissociation products of such excited molecules can include energetic neutrals, whose high kinetic energies are accounted for by the Franck-Condon principle. These Franck-Condon neutrals have effective mean free paths which can be of the order of the plasma dimensions and unlike the cold neutrals can penetrate into the plasma volume. It was conservatively assumed for these calculations that every ionization of a cold neutral molecule at the plasma surface produces a Franck-Condon neutral atom which has a probability $\frac{1}{2}$ of entering the plasma.

Since the relative speed of the interacting particles is essentially the speed of the plasma ions, the plasma loss rate by charge exchange with background neutrals is approximated by the term

$$
\left(\frac{\partial g}{\partial t}\right)_{c \times 0}=\left\langle\sigma_{c x} v\right\rangle\left(n_{0}+n_{f}\right) g(v, t)
$$

$<\sigma_{c x} v>$ is conservatively approximated by $\sigma_{c x}=10^{-15} \mathrm{~cm}^{2}$, multiplied by the ion speed $v$. The quantities $n_{0}$ and $n_{f}$ are the exfective densities of cold neutrals and Franck-Condon neutrals in the plasma volume and must be calculated self-consistently as the plasma density changes. The effective mean density $n_{0}$ of cold neutrals in the plasma is obtained from the rate equation:

$$
\frac{d n_{0}}{d t}=\frac{N_{0} v_{0}}{4} \frac{S}{v}-\frac{n_{0} v_{0}}{4} \frac{S}{v}-\left\langle\left(\sigma_{i}+\sigma_{c x}\right) v\right\rangle n_{0}
$$

The first two terms represent the influx of cold neutrals of ambient density $\mathrm{N}$ into the plasma surface area $S$ and the leakage out of the plasma volume. The third term is the rate of destruction of cold neutrals in a plasma of density $n$ by ionization and charge exchange. In the GAIN code, $\mathrm{n}_{0}$ is evaluated as the formal solution of Eq. 10 in the form

$$
\begin{aligned}
n_{0}(t) & =N_{0} \exp \left\{-\int_{0}^{t}\left[\frac{v_{0} S}{4 V}+\left\langle\left(\sigma_{i}+\sigma_{c x}\right) v\right\rangle n\left(t^{\prime}\right)\right] d t^{\prime}\right\} \\
x & \left\{1+\frac{v_{0} S}{4 V} \int_{0}^{t} \exp \left\{\int_{0}^{t^{\prime}}\left[\frac{v_{0} S}{4 V}+\left\langle\left(\sigma_{i}+\sigma_{c x}\right) v\right\rangle n\left(t^{\prime \prime}\right)\right] d t^{\prime \prime}\right\} d t^{\prime}\right\}
\end{aligned}
$$


The effective mean density of Franck-Condon neutrals is determined from the influx of these particles and from their escape probability. The charge exchange rate, $<\sigma_{\mathrm{cx}} \mathrm{v}>\mathrm{nn} \cdot \mathrm{f}^{\prime}$ is equated to the product of the current of particles into the plasma surface, ' $\frac{1}{2} \frac{0{ }^{\circ}}{4}$ and $1-P$, where $P$ is the probability of escape without charge exchange approximated by $(\operatorname{Ref} .5) P=\left(1+\bar{l} / \lambda^{\prime}\right)^{-1}$. Here, $\lambda^{\prime}$ is the effective mean free path $\lambda^{\prime}=v_{f} / n<\sigma_{c x} v>$ while $\bar{l}$ is the mean chord length through the plasma, $\bar{l} \approx 4 \mathrm{~V} / \mathrm{s}$. The density of Franck-Condon neutrals is, therefore, calculated in the GAIN code as

$$
n_{f}(t)=\frac{1}{2} \frac{N_{0} v_{0}}{v_{f}}\left\{1+\frac{4 V}{S} \frac{\left\langle\sigma_{c x} v\right.}{v_{f}} n(t)\right\}^{-1}
$$
The charge exchange cross-section is approximated by a constant $\sigma_{\mathrm{cx}}=10^{-15} \mathrm{~cm}^{2} \mathrm{so}$
that

$$
\left\langle\sigma_{c x} v\right\rangle_{n}=4 \pi \sigma_{c x} \int_{0}^{\infty} g(v, t) v^{3} d v
$$

The characteristic speed $v_{f}$ of Franck-Condon particles was chosen as $8 \times 10^{5} \mathrm{~cm} / \mathrm{sec}$. It was assumed that after escape from the plasma a Franck-Condon neutral becomes a cold neutral upon contact with the wall of the vacuum chamber. On this assumption, there is no build-up of Franck-Condon neutrals in the vacuum system.

\section{b. Target Plasma Time History}

For calculations of the time history of a dense laser-produced plasma used as the target for an energetic neytral injection beam, the plasma surface area $S$ and volume $V$ were chosen as $189 \mathrm{~cm}^{2}$ and $95 \mathrm{~cm}^{3}$, respectively, commensurate with the field length and plasma volume anticipated in the UARL mirror experiments. The transverse diameter of an elliptical plasma with this surface area and volume would be about $2 \mathrm{~cm}$, which was taken as the mean chord length $\mathrm{L}$ of Eq. 8. The plasma ions and electrons were assumed initially Maxwellian at a temperature of $500 \mathrm{eV}$, with equal densities of $5 \times 10^{15^{-3}} \mathrm{~cm}^{-3}$. The cold background gas neutral speed was chosen as $v_{0}=4 \times 10^{4} \mathrm{~cm} / \mathrm{sec}$, characteristic of nitrogen at room temperature, while the Franck-Condon speed was $8 \times 10^{5} \mathrm{~cm} / \mathrm{sec}$, characteristic of nitrogen atoms with kinetic energies of several electron volts. In the case discussed here, the beam current incident on the plasma was $100 \mathrm{~mA}$ equivalent atoms in an energy distribution with a maximum at $10 \mathrm{keV}$ and full width at half-intensity of $2 \mathrm{keV}$. These plasma and beam parameters were considered typical of what could be achieved in the laser initiated target experiment (LITE) in the $\mathrm{R}=2$ minimum-B field of the UARL baseball coil.

Using the modified GATN code described in the previous section, calculations were made of the target plasma evolution with the incident energetic neutral beam. The time history of the electron and ion temperature, plasma potential, and plasma density is shown in Fig. 1. For the first millisecond, the plasma behavior has the same time dependence as the decay calculated in the absence of a beam. As charge exchange with 
the beam atoms replaces the low energy plasma ions by $10 \mathrm{keV}$ ions, the ion temperature rises and, with it, the electron temperature and plasma potential. Although the ionization electrons are introduced into the plasma with energies of about $5 \mathrm{eV}$, they are rapidly heated by collisions with the ions and other electrons. The plasma potential is a parameter adjusted to equalize the ion and electron loss rates by the trapping of lower energy electrons. As the electron temperature rises, the potential also increases, in order to limit the electron loss rate to the ion loss rate.

The initial density of cold neutrals in the plasma volume was chosen equal to the ambient density $\mathrm{N}_{0^{\circ}}$ The cold neutral density is rapidly (e-folding time $\sim 5 \mathrm{nsec}$ ) reduced by ionization and charge exchange to a value, $n_{0}$, several orders of magnitude smaller than $\mathbb{N}_{0}$. After this initial transient, the time derivative in $\mathrm{Eq}$. 10 is much smaller than the other terms and the density calculated by the GAIN code is well approximated by

$$
n_{0}(t)=\frac{N_{0} v_{0}}{4} \frac{S}{v}\left\{\frac{v_{0} S}{4 v}+\left\langle\left(\sigma_{i}+\sigma_{c x}\right) v\right\rangle n\right\}^{-1}
$$

The calculated mean density of Franck-Condon neutrals is about a factor of two smaller than $\mathrm{n}$, as may be seen by comparison of Eqs. 12 and 14 upon noting that in each case the second term in the brackets is small compared with the first. The plasma losses by charge exchange with Franck-Condon neutrals were, therefore, always less than those caused by charge exchange with cold neutrals. For a neutral background density $N_{0}=10^{11} \mathrm{~cm}^{-3}$, Fig. I shows a precipitous loss of plasma after the plasma density falls below $10^{13} \mathrm{~cm}^{-3}$. In accordance with Eq. 14, the cold neutral density in the plasma volume rises rapidly to its ambient value, $N_{0}$, as the plasma is destroyed by charge exchange. For $\mathrm{N}=10^{10} \mathrm{~cm}^{-3}$, the plasma lasts for more than 10 milliseconds, while for $\mathrm{N}_{0}=10^{9} \mathrm{~cm}^{-30}$, the plasma lifetime is about 100 milliseconds. For a background density of $10^{8} \mathrm{~cm}^{-3}$, the plasma survives losses by charge exchange with background neutrals, and the density decay is halted and subsequently built up by ionization of the neutral beam to a steady state value of $3 \times 10^{12} \mathrm{~cm}^{-3}$. In the steady state, the cold neutral density in the plasma volume is reduced below its ambient value $\mathrm{N}_{0}$ by a factor of 30 . The steady-state ion temperature levels off at about $10 \mathrm{keV}$, the energy of the injected neutrals, while the electron temperature, which continues to increase slowly, is about one-tenth of the ion temperature.

The time evolution of the electron and ion distribution functions are shown in Fig. 2. It may be noted that electron distribution functions at times .0003 sec and $.0043 \mathrm{sec}$ are similar but shifted on the vertical logarithmic scale. This is consistent with the virtually constant electron temperature shown in Fig. 1 for the same interval of time. In this same time interval, the ion distribution tends to spread in the direction of larger values of ion speed as the result of charge exchange with the neutral beam. The peak intensity of the neutral beam occurs at a speed of $1.4 \times 10^{8} \mathrm{~cm} / \mathrm{sec}$, and the bump which develops on the upper edge of the ion distribution corresponds to ions introduced by charge exchange and ionization of the beam. These ions merge with the distribution function in such a way that $\partial \mathrm{g} / \partial \mathrm{v}$ is always negative. The ion distribution labeled 1.846 seconds is a steadystate distribution. The electron distribution, on the other hand, continues to 
shift slowly toward larger speeds after 1.846 seconds, as the electrons continue to be heated by collisions with the ions.

\section{Beam and Vacuum Requirements}

From the results of the Fokker-Planck calculations presented in Fig. 1, the vacuum background gas density has a profound influence on the steady-state target plasma density sustained by neutral beam injection. For a given value of injected beam current there is some maximum value of background gas density, between $10^{8} \mathrm{~cm}^{-3}$ and $10^{9} \mathrm{~cm}^{-3}$, for which a steady state is possible for the injection and containment conditions considered in these calculations. Conversely, if the background density is fixed, a minimum injected beam current is required for the existence of a steady state. In this section, the relationship between the required neutral beam current and permissible background gas density are examined as a function of beam and containment parameters.

An equation for the time-dependent plasma ion density may be obtained by multiplying Eq. 2 by $4 \pi v^{2}$ and integrating over $v$. Since elastic collisions conserve particles, the integral of the Fokker-Planck term vanishes identically. Charge exchange with the neutral beam also conserves plasma particles and the integrals of the last two charge exchange terms in Eq. 2 cancel. From the remaining terms a rate equation for the plasma ion density is obtained as

$$
\frac{d n}{d t}=\frac{I L}{v} n\left(\sigma_{e}+\sigma_{i}\right)-\frac{\lambda}{3} \Gamma v^{-3} n^{2}-\sigma_{c x} v\left(n_{0}+n_{f}\right)
$$

in which $v$, the characteristic speed for the ion distribution function, is in general time-dependent. For the steady state, if one exists, the plasma density is obtained as a function of the steady-state value of $v$ and the densities $n_{0}$ and $n_{f}$ by setting the time derivative in Eq. 15 equal to zero. The neutral densities $n_{f}$ and $n_{0}$ are obtained from Eqs. 12 and 14, so that $n$ may be written as an implicit function of beam current $I$ and background density $N_{0}$, namely,

$$
0=\frac{I L}{V}\left(\sigma_{e}+\sigma_{i}\right)-\frac{\lambda}{3} \Gamma v^{-3} n-\sigma_{c x} v N_{0}\left\{\frac{1}{1+\frac{4 V}{S}\left(\sigma_{i}+\sigma_{c x}\right) \frac{v}{v_{0}} n}+\frac{v_{0} / 2 v_{f}}{1+\frac{4 V}{S} \sigma_{c x} \frac{v}{v_{f}} n}\right\}
$$

Here, $\lambda$ is the lowest eigenvalue for the loss cone angular distribution which, for a mirror ratio $R=2$, is $\lambda=3.32$. The parameter $\Gamma$ is a collection of constants in the Coulomb cross-section evaluated here as $2 \times 10^{12}$. In Fig. 3, the ion density, computed from Eq. 16 for the parameter values listed in the following table, is plotted against equivalent beam current in amperes $\left(l=I / 6 \times 10^{18}\right)$. 
Table I.

$$
\begin{aligned}
& \mathrm{L}=2 \mathrm{~cm} \\
& \mathrm{~V}=94.5 \mathrm{~cm}^{3} \\
& \mathrm{~S}=189 \mathrm{~cm}^{2}
\end{aligned}
$$

$$
\begin{aligned}
v & =1.5 \times 10^{8} \mathrm{~cm} / \mathrm{sec} \\
v_{0} & =4 \times 10^{4} \mathrm{~cm} / \mathrm{sec} \\
v_{f} & =8 \times 10^{5} \mathrm{~cm} / \mathrm{sec} \\
N_{0} & =10^{8} \mathrm{~cm}^{-3}
\end{aligned}
$$

$$
\begin{aligned}
& \sigma_{e}+\sigma_{i}=2 \times 10^{-16} \mathrm{~cm}^{2} \\
& \sigma_{c x}=10^{-15} \mathrm{~cm}^{2} \\
& \sigma_{i}+\sigma_{c x}=1.6 \times 10^{-15} \mathrm{~cm}^{2}
\end{aligned}
$$

The steady-state density of $3 \times 10^{12} \mathrm{~cm}^{-3}$, obtained from the Fokker-Planck calculation for $\mathrm{N}_{0}=10^{8} \mathrm{~cm}^{-3}$ and $l=0.1$ ampere, is indicated as the circle on the upper branch of the curve. The neutral density $n$ associated with the different $n(l)$ values was calculated from Eq. 14 and is plotted against beam current on the right of Fig. 3 . The upper branch of the $n$ curve corresponds to the lower branch of the $n$ curve and vice versa. Thus the end point of the $n_{0}$ curve at $10^{8} \mathrm{~cm}^{-3}$ corresponds to $n=0$. The minimum injected beam current required for a steady state with a background density of $N_{0}=10^{8} \mathrm{~cm}^{-3}$ is seen to be about 80 milliamperes. A similar set of curves has previously been presented in Ref. 6 . In that report, the effects of electrons on the ion distribution were not considered, Franck-Condon neutrals were neglected, and some of the parameters listed in Table I were assigned other values. Even so, the calculated minimum value of injected beam current, 50 ma, was not greatly different from that obtained by the present more complete analysis. If $N_{0}$ is varied while the other parameters of Table I are held constant, the family of ${ }^{\circ}$ curves of plasma density versus beam current appear as shown in Fig. 4. The upper branch of each such curve is asymptotic to the straight line obtained by setting $\mathrm{N}=0$ in $\mathrm{Eq}$. 16. It may be shown that the minimum current necessary for a steady state varies approximately as $N^{\frac{1}{2}}$. On the other hand, the value of the current at which the plasma density goes to zero on the lower branch, the "corner current", is proportional to $\mathrm{N}$, as may be found from setting $\mathrm{n}=0$ in $\mathrm{Eq}$. 16. The corner current represents an upper bound to the beam current required to achieve the transition to the upper branch of the $n$ vs. l curve starting from Lorentz ionization buildup. Inspection of Eq. 16 also reveals that the effects of increasing the mirror ratio ( $\operatorname{smaller} \lambda$ ) or increasing plasma ion temperature (larger $v$ ) are to reduce the injected beam current required to sustain a specified plasma density, i.e., the line asymptotic to the upper branch of the ion density curves is shifted to the left by these changes. The effect of the plasma shape on the beam current requirement is also apparent from Eq. 16. We note that, for constant plasma volume, a transition from the elongated ellipsoid assumed in the present calculations to a more spherical shape results in an increased mean chord length, $L$, and a reduced surface area $S$. Both changes have the effect of reducing the required beam current. 


\section{B. Mirror Plasma Confinement}

\section{Mirror-Confined Plasma Decay}

Analysis of the collisional decay of a mirror-confined plasma has brought to light a class of solutions corresponding to sudden, rapid loss of the plasma. This rapid decay is unrelated to plasma-wave instabilities, but arises from the dependence of the plasma loss rate on the ion energy $\left(E_{i}-3 / 2\right)$.

By taking velocity moments of the Fokker-Planck equations, Eqs. 1 and 2, but neglecting the source and charge exchange terms, equations are obtained describing the decay of a mirror-confined plasma dominated by Coulomb scattering into the loss cones. The distribution-averaged ion speed is much smaller than the average electron speed and, by neglecting terms of order of that ratio, a set of relatively uncluttered rate equations is obtained for the plasma density and the mean energies of the ions and electrons:

$$
\begin{aligned}
& \frac{d n}{d t}=-A n^{2} E_{i}^{-3 / 2} \\
& \frac{d E_{i}}{d t}=A\left(1-c_{i}\right) n E_{i}^{-1 / 2}=B\left(E_{i}-E_{e}\right) E_{e}^{-3 / 2} n \\
& \frac{d E_{e}}{d t}=A\left(1-c_{e}\right) n E_{e} E_{i}^{-3 / 2}+B\left(E_{i}-E_{e}\right) E_{e}^{-3 / 2} n
\end{aligned}
$$

The constants $A$ and $B$ are collections of physical parameters, namely,

$$
A=\frac{\lambda b}{3 m_{i}^{1 / 2}} 4 \pi e^{4} \ln \Lambda, \quad B=a \frac{m_{e}^{1 / 2}}{m_{i}} 4 \pi e^{4} \ln \Lambda
$$

The coefficients $\mathrm{a}$ and $\mathrm{b}$ are dependent on the shape of the distribution function, but not sensitively, and are approximately equal to 1.5. The quantity $\lambda$ is the eigenvalue for the lowest order mode of the loss cone angular distribution while $\ln \Lambda$ is the familiar Coulomb logarithm. The parameter $\mathrm{C}_{i}$ is the reciprocal of a product of velocity averages over the ion distribution function, i.e.

$$
c_{i}^{-1}=\left\langle v^{-2}\right\rangle_{i}\left\langle v^{2}\right\rangle_{i}
$$

For a loss cone distribution of ions of the form $v^{m} e^{-\beta v^{2}}$,

$$
c_{i}=\frac{m+1}{m+3}
$$

which ranges from $1 / 3$ for very small $\mathrm{m}$, to unity for large $\mathrm{m}$. For a mirror ratio of $2, \mathrm{~m}$ is nearly unity, and $\mathrm{C}_{i}$ is about $1 / 2$. The parameter $\mathrm{C}_{\mathrm{e}}$ depends on averages over the electron dictribution function, and is defined as

$$
c_{e}^{-1}=\left\langle v^{-2}\right\rangle_{e}^{c}\left\langle v^{2}\right\rangle_{e}
$$


Whereas $\left\langle v^{2}\right\rangle$ is an average over the entire electron distribution, $\left\langle v^{-2}\right\rangle^{c}$ is an average over only that part of the distribution in which the electrons have energies larger than the plasma potential energy e $\varphi$. Thus $\mathrm{C}_{e}$ is the ratio of some multiple of $e_{\varphi}$ to $E_{e}$ and is generally considerably larger than unity.

The first term in each energy equation gives the rate of change in particle energy resulting from scattering into the loss cones - positive for ions, but negative for electrons. The second term in each of these equations gives the rate of energy transfer between the ions and electrons. If the electrons are much colder than the ions, they rapidly drain off energy from the ions, enhancing the plasma loss rate predicted by Eq. 17. A particular solution of these rate equations (Eqs. 17-19) has a constant ratio $\mathrm{E}_{i} / \mathrm{E}_{\mathrm{e}}$, given in terms of the other constants by

$$
\left(\frac{E_{i}}{E_{e}}\right)^{5 / 2}-\left(\frac{E_{i}}{E_{e}}\right)^{1 / 2}=\left(c_{e}-c_{i}\right) A / B
$$

The solutions of the energy equations satisfy this relation after passing through an initial transient during which the plasma potential undergoes a rapid adjustment for equalization of the electron and ion loss rates. Let $t$ be the time at which $\mathrm{Eg} \cdot 21$ first applies and $n(t), E$
Upon combining Eqs. 19 and 18 , a solution is obtained for the ratio

$$
\frac{E_{i}^{3 / 2}}{n}=\frac{E_{i 0}^{3 / 2}}{n_{0}}\left(1+c\left(t-t_{0}\right)\right)
$$

in which

$$
c=n_{0} D / E_{i 0}^{3 / 2}
$$

and

$$
D=\left(\frac{5}{2}-\frac{3}{2} c_{i}\right) A-\frac{3}{2} B\left\{\left(\frac{E_{i o}}{E_{e o}}\right)^{3 / 2}-\left(\frac{E_{i 0}}{E_{e o}}\right)^{1 / 2}\right\}
$$

Substitution of this solution into Eqs. 17-19 gives for $D \neq 0$,

$$
\begin{aligned}
& n(t)=n_{0}\left(1+c\left(t-t_{0}\right)\right)^{-\alpha} \\
& E_{i}(t)=E_{i 0}\left(1+c\left(t-t_{0}\right)\right)^{2(1-\alpha) / 3}
\end{aligned}
$$

and a corresponding expression for $E_{e}(t)$.

The exponent $\alpha$ is

$$
\alpha=\frac{A}{D}=\frac{A n_{0}}{c E_{i 0}{ }^{3 / 2}}
$$


For "classical" decay, D and, therefore $\mathrm{C}$ and $\alpha$, are all positive. When $\alpha=1$, $C$ may be identified with the collision frequency for scattering into the loss cones, the ion and electron temperatures are time-independent, and the plasma density decays as $t^{-1}$ for large values of $C t$. If $A$ is larger than $D, \alpha$ is larger than unity, the ion energy decreases with time, the collision frequency increases, and the plasma decay goes faster than $t^{-1}$. For sufficiently large values of $\mathrm{C}, \mathrm{Eq} .21$ implies that $E_{i} / E_{\text {m }}$ may be large enough that $D$ becomes negative in $\mathrm{Eq} .24$. In this case, both $\mathrm{C}^{\mathrm{i}}$ and $\alpha$ must be negative, and the solutions for the plasma density and particle energies of Eqs. 25 go to zero at a time $t=t_{0}+|C|^{-1}$, corresponding to a sudden rapid loss of plasma. For values of $t$ such that $|c|\left(t-t_{0}\right) \ll 1$, this plasma behavior may, however, be indistinguishable from "classical decay" and the later sudden loss of plasma mistakenly attributed to plasma-wave instabilities. For the case in which $D$ is zero, the time dependence of the plasma density and particle energies becomes exponential. Solutions for this case are included here for the sake of completeness:

$$
\begin{aligned}
& n(t)=n_{0} \exp \left(-\beta\left(t-t_{0}\right)\right) \\
& E_{i}(t)=E_{i 0} \exp \left(-\frac{2}{3} \beta\left(t-t_{0}\right)\right)
\end{aligned}
$$

in which

$$
\beta=A n_{0} / E_{i 0}^{3 / 2}
$$

Results of Fokker-Planck calculations of plasma decay in the minimum-B field of the UARL baseball coil, without neutral injection or charge exchange effects, yield time-dependent ion and electron energies in the constant ratio $\mathrm{E}_{\mathrm{i}} / \mathrm{E}_{\mathrm{e}}=6.9$. $\mathrm{A}$ ratio of 14 is required for $D$ to vanish in Eq. 24, and still larger ratios are required for negative $\mathrm{D}$. We should, therefore, not expect to observe in the UARL experiments the type of plasma decay characterized by negative values of $\mathrm{C}$ and $\alpha$.

\section{Length and Radial Size Stability Criteria}

The stability of the dense (finite $\beta$ ) plasmas confined in the UARL baseball coil mirror magnetic containment field has been analytically examined to determine the critical radial size of the plasma against flute-like $\left(k_{1}=0\right)$ drift loss cone modes and the critical axial length for a number of potentially dangerous microinstabilities.

Using the theory of Tang, Pearlstein, and Berk (Ref. 7), extensive computer calculations were performed to evaluate the critical radius for development of the flute-like drift loss cone modes as a function of the plasma $\beta$ for different values of plasma electron and ion temperatures. Figure 5 gives the results obtained for a hydrogen plasma with a mirror ratio of two and an ion to electron temperature ratio of five for ion temperatures over the range .2 to $10 \mathrm{keV}$. The critical radius is plotted as the ratio $R_{p} / a_{i}$, where $a_{i}$ is the ion Larmor radius, and for a given set of piasma $\beta$ and temperature conditions, plasmas larger than $R$ in radius will be stable. The strong stabilizing effect of finite $\beta$ ( $R$ decreasing with $\beta$ ) for this instability is evident from the curves. In addition, the critical radius is observed 
to be a sensitive function of the ion temperature at low $\beta$, but is nearly independent of the ion temperature at larger $\beta$. For $\beta>0.3$, a factor of fifty change in the ion temperature causes only a $5 \%$ decrease in the critical radius, while the same change in ion temperature at $\beta \sim 10^{-3}$ causes the critical radius to decrease by more than a factor of seven. Further calculations not shown in the figure established that the critical radius for the drift loss cone mode is essentially independent of changes in the electron temperature. The calculations of Fig. 5 are based on an infinite medium approximation and give an upper bound for the critical radius. In Ref. 7, Tang et. al. also consider the stabilizing influence of finite geometry. The finite geometry effects decrease the value of the critical radius, but qualitatively there is very little difference from the infinite medium results of Fig. 5 . Of particular interest for the UARL mirror confined laser produced target plasma experiments is the observation that the critical radius at $\beta \sim 10^{-3}$ and $\mathrm{T}_{i}=10 \mathrm{keV}$ is less than the critical radius at $\beta \sim 1.0$ for $T$ in the 1 to $10 \mathrm{keV}$ range. As a result, a plasma which is initially captured at $\beta \sim 1.0, \mathrm{~T}_{j}=1.0 \mathrm{keV}$ will become even more stable against the flute-like drift cone modes after the ion temperature is increased to $10 \mathrm{keV}$ by the injection of energetic neutral particles into the lower B mirror confined target plasma.

The critical length for plasma stability has been examined for the negative energy flute modes, the convective loss cone modes, and the non-flute drift cone and negative energy instabilities. Convective modes will exist in a plasma of a sufficient length to contain several "e folding" distances. In the case of an absolute instability, the plasma must be at least as long as the wavelength of the instability. Hence, the critical length determines the maximum plasma length for which the stability can be maintained. Tang and co-workers (Ref. 7) give the criteria for stability against negative energy flute modes as

$$
L / a_{i}<3\left[\frac{2\left(R^{2}-1\right)}{R}\right]^{1 / 2} \beta^{9 / 8}\left\{\left(\frac{c}{2 \pi}\right)^{2} \cdot\left[\left(\frac{\omega_{c i}}{\omega_{p i}}\right)^{2}+\frac{m}{M}\right]\right\}^{-3 / 8}
$$

where $R=$ mirror ratio, $m$ and $M$ are the electron and ion masses, $C=$ $2 \sqrt{\pi}(R+I)^{3 / 2} /\left(R+R^{\frac{1}{2}}\right)$, and $\omega_{c i}$ and $\omega_{p i}$ are the ion gyrofrequency and ion plasma frequency respectively. Using equation 28 , the value of $\mathrm{L} / \mathrm{a}_{i}$ for a plasma with mirror ratio of two, ranges from 100 for $\beta \sim 1.0$ to about 5 for $\beta \leq$. 1 . The stabilizing effect of finite $\beta$ is evident in the form of $\mathrm{Eq} .28$.

Post and Rosenbluth (Ref. 8) consider the length criterion for stability of collisional plasmas against the convective loss cone mode in an infinite, low $\beta$ plasma. Using the parameters for the plasma in the UARL baseball system, the critical length for stability determined from their analysis is $\mathrm{L} / \mathrm{a}_{i} \leq 100$. Berk, Pearlstein, and Cordey (Ref. 9) in a more recent publication include the effects of axial variation of the magnetic field and finite $\beta$ and give the critical length for the convective loss cone mode as well as the non-flute $\left(k_{11} \neq 0\right)$ drift cone and negative energy modes as 


$$
L / a_{i} \lesssim\left[\frac{M}{m N^{2}\left(k_{\perp} a_{i}\right)^{2} F_{N}}\right]^{1 / 2}\left[R\left(1+\frac{\omega_{p e} e^{2}}{k_{\perp}^{2} c^{2}}\right)\right]^{-1 / 2}
$$

where $\mathrm{N}$ = ion cyclotron harmonic number

$$
F_{N}=\frac{2 \omega_{c i}^{2}}{k_{\perp}^{2}} \int d^{3} v \frac{\partial f}{\partial v_{\perp}^{2}} J_{n}^{2}\left(\frac{k_{\perp} v_{\perp}}{\omega_{c i}}\right)
$$

The critical lengths calculated from Eq. 29 are somewhat more restrictive than those of Post and Rosenbluth. For instabilities near the ion cyclotron frequency and a mirror ratio of two, Eq. 29 gives the critical length as $L / a \sim 60$ for $8 \sim$ 1.0. The length increases as $\beta$ decreases, to a limiting value of $\dot{t} / a_{i} \sim 120$ for zero $\beta$. It is significant to note that finite $\beta$, which has a stabilizing effect on the critical size for the flute-like microinstability modes, is a destabilizing influence when wave propagation is permitted parallel to the applied magnetic field $\left(k_{11} \neq 0\right)$.

The projected size of the target plasma in the UARL baseball system is about 30 ion larmor radii long and 5 to 10 ion larmor radii in radius. Hence, the convective loss cone and the $\mathrm{k}_{11} \neq 0$ drift cone mode are not expected to occur in our system. However, the flute-like negative energy mode in the plasma's lower $\beta$ mirror confined stage as well as the flute-like drift cone modes may occur.

Because of the possible concern with the flute-like drift cone mode, a more detailed study of the drift cone instability is proceeding to determine the dispersion characteristics of both the non-flute and the flute-like modes in the finite $\beta$ limit. The general dispersion relation including finite $\beta$ effects is being developed from the linearized Vlasov equation and Maxwell's equations. The loss cone distribution of Dory, Guest, and Harris (Ref. 10) and an infinite medium approximation are being used. From the progress made to date, extensive computer calculations will be required in order to solve the dispersion relation, and attempts to put the equation in a more tractable form are continuing. The equation for the flute-like modes is less complex than the general relation, and the emphasis on the numerical calculations will be placed on these modes because of their possible occurrence in the UARL plasma system. 


\section{EXPERTMENTAL PROGRAM}

\section{A. Diagnostic Development}

\section{Laser Interferometer Diagnostics}

The initial capture and transition to mirror confinement of $500 \mathrm{ev} \mathrm{-} \mathrm{I} \mathrm{keV}$ plasmas in a 10-15 kG magnetic containment field occurs at a plasma density of $10^{15}-10^{16} \mathrm{~cm}^{-3}$. To measure the plasma density decay of these confined plasmas down to the $3 \times 1013 \mathrm{~cm}-3$ cut-off density of the $6 \mathrm{~mm}$ microwave interferometer diagnostic, a.fractional fringe $\mathrm{CO}_{2}$ laser interferometer operating at $10.6 \mu$ has been developed. Since the characteristic time for the magnetically confined laserproduced plasma to decay to $1013 \mathrm{~cm}^{-3}$ after capture by the magnetic field is on the order of 10-100 $\mu \mathrm{sec}$, the laser interferometer must possess a temporal resolution capability of $I \mu \mathrm{sec}$ or less. However, the use of conventional laser interferometers (Refs. 11 and 12) to measure the average electron area density, $\left\langle n_{e}>l_{p}\right.$, of temporally varying plasmas is generally limited to characteristic plasma time variations greater than $10 \mu \mathrm{sec}$. This time response is determined by the $Q$ of the laser cavity, the gain of the laser discharge, and the lifetimes of the collisional processes contributing to the laser transition. A novel technique for circumventing this time response limitation without compromising the high spatial resolution of conventional laser interferometry is to locate the plasma under investigation in one or more arms of a transmission ring resonator excited by an external laser. With this system the phase shift per pass of the radiation circulating in the ring changes as the plasma density varies. The interference of this circulating radiation with the radiation injected into the ring resonator from the externally positioned laser alters the intensity of the radiation reflected from or transmitted through the ring resonator, either of which may be taken as the output signal as shown in Fig. 6 . The operation of a transmission ring resonator is equivalent to that of a linear resonator with curved or plane mirrors and focusing elements (Ref. 13), and the interpretation of its output signal is similar to that described previously for conventional laser interferometers. By using a ring resonator the laser is decoupled from the reference cavity containing the plasma without the use of separate isolation elements which at $10.6 \mu$ lie near the edge of current technology and have only a limited rejection ratio. With a ring resonator cavity the response time is determined only by the $Q$ of the resonator which may be varied by changing the reflectivities, losses, and, separation of the cavity mirrors. Thus the transmission ring resonator significantly improves the time response of the laser interferometry without incurring a loss in sensitivity or spatial resolution.

This technique has been verified using a three mirror $\mathrm{CO}_{2}$ laser excited transmission ring resonator to measure the afterglow plasma density decay of a pulsed helium discharge. A fractional fringe detection capability was achieved by modulating the perimeter of the ring resonator with a mirror modulator consisting of an $x$-cut, quartz peizoelectric disk, gold coated on both surfaces for maximum 
reflectivity and held at three points on the circumference midway between the two surfaces. A new high gain I to $E$ converter developed by the UARL Electronics Instrumentation Group was used to process the interferometer output signal of the Ge:Au detector. Using this amplifier the detection system bandwidth is $500 \mathrm{~Hz}$ to $5 \mathrm{MHz}$. The low frequency roll-off suppresses the deleterious effects of random laboratory noise.

Preliminary design of a four mirror resonator system for installation onto the experimental chamber has been completed and is shown in Fig. 7 . In the design of the individual mirror mounts careful attention has been given to the requirements of rigidity, to avoid spurious noise in the resonator output, and high vacuum while maintaining the capability of external optical alignment. Experimental bench tests of this configuration with the new amplifier indicate that it is capable of a 10-3 fractional fringe detection capability, permitting measurements down to densities of $3 \times 10^{13} \mathrm{~cm}-3$, the upper limit of the $6 \mathrm{~mm}$ microwave system.

\section{R.F. Emission Diagnostics}

Two microinstabilities, the convective loss cone and the resonant drift cone instability, although not observed in the earlier investigations, may cause rapid loss of plasma on the longer time scale of the current confinement experiments. The resonant drift cone modes discussed earlier, as well as enhanced particle scattering resulting from a partially developed convective loss cone instability (Ref.14) could result in particle loss on a time scale shorter than that due to classical Coulomb scatter into the mirror loss cones. The characteristic frequencies associated with the appearance of these instabilities are distinctly different for the plasma conditions of the present experiments; at harmonics of the ion cyclotron frequency which are in the rf range for the drift cone instability (Ref. 8) and in the vicinity of the ion plasma frequency which is in the microwave range for the convective loss cone instability (Refs. 8 and 15). To test for $\mathrm{rf}$ emission accompanying the plasma decay, a simple antenna consisting of two pairs of loop antennas (Ref.16) oriented in two perpendicular planes has been constructed, Fig. 8 . A balanced electrostatic shield is employed to achieve cancellation of the effects of currents induced by radial electric fields. In order to minimize the generation of spurious signals, the resistance of the wire used in the construction of each loop was chosen so that the input impedance of each loop is nominally 50 ohms matching the impedance of the coaxial cable used to transmit the signal from each loop. The signals from each pair of loops are differentially amplified using a Tektronics IA5-585A plug-in unit and oscilloscope combination to complete the cancellation of electrostatic components. The antenna loops are oriented so that one pair of loops is aligned for maximum sensitivity to the axial and the other to the azimuthal rf magnetic field component radiated by the confined plasma. The sensitivity of this rf probe was verified by observing the turn-on and decay of the main magnetic field. Observations with this probe will allow detection of rf energy from the plasma over a broad band of frequencies, $0-50 \mathrm{MHz}$, including the ion cyclotron frequency ( 15 $\mathrm{MHz} @ 10 \mathrm{kG}$ ) and its higher harmonics. Such signals correlated in time with the 
plasma density decay as measured by the $\mathrm{CO}_{2}$ and microwave interferometers should provide information regarding the presence or absence of the drift cone instability.

\section{B. Experimental Progress}

\section{System Operation}

Operation of the major components and of the complete experimental system for investigation of initial capture, mirror confinement, and subsequent decay of laserproduced plasmas in minimum-B mirror magnetic fields has, during this contract period, been devoted in the main to the integration of components and to the development and evaluation of operating procedures. Although a number of electronic and vacuum difficulties were encountered and corrected and several improvements in the magnet cooling and in the LiH particle suspension system were instituted, no major problems were encountered either in the magnet system itself or in incorporating the operation of the magnet with the complete system.

The minimum-B magnet used in these experiments, described in detail in Ref. $I$, is of spherical, baseball-sealn geometry with a maximum center field intensity of $15 \mathrm{kG}$, mirror ratio and well depth of 2, and distance between mirror points of 31 $\mathrm{cm}$. The coil windings are copper, pre-chilled with liquid nitrogen to reduce the coil resistance and keep the power requirements within the current and voltage limits of the power source for the required maximum testing time of $1-2 \mathrm{sec}$. In the first cooling tests of the magnet, approximately 100 hours were required to chill the entire magnet (which consists of $\sim 500 \mathrm{lbs}$ of stainless steel and $\sim 250$ lbs of copper windings) from room temperature to liquid nitrogen temperature. With improved liquid transfer line insulation and a higher liquid dewar pressure (48 psi) to increase the flow rate, the room temperature to liquid nitrogen temperature chill time has been reduced to about 40 hours. Since only the copper windings are heated during the magnet current pulse, the cooling rate between successive current pulses is very much larger than the initial chilling rate, and, for the present experiments in which the required current pulse curation is $<0.5 \mathrm{sec}$, the repetition rate for experiments is not limited by magnet recooling time for field intensities < $10 \mathrm{kG}$. Even at the maximum field intensity of $15 \mathrm{kG}$, the recooling time should not exceed 30 minutes with the existing cooling rates, and even greater cooling rates are available, if required, by increasing the dewar pressure to 80-90 psi.

The magnet has been operated satisfactorily at magnet currents up to 1150 amp (8.4 $\mathrm{kG}$ center field intensity), the maximum current runs undertaken to date. In addition, the complete servo control system for maintaining constant current in the coil, described in Ref. 17, was found to function as designed. As shown in Fig. 9, the servo system held a current of $1000 \mathrm{amp}$ within 0.5 percent for a constant current duration of $I \mathrm{sec}$. It is also apparent from this data that the servo system provides the additional advantage of an effectively shorter current rise time by eliminating the exponential tail of the current rise. This can provide a significant reduction in the overall duration of the current pulse and, consequently, less joule heating of the magnet coil for a given testing time at constant current, 
particularly important for the higher current operation.

In the operation of the complete experimental system, the generation of the laser-produced plasma must be delayed in time until the magnetic field has attained its steady-state value. Since the magnet current rise time is relatively long ( $\leq 0.25 \mathrm{sec})$ compared to the other events in the operating sequence, a signal derived from the amplitude of the magnet current is used to start the entire sequence. This means that the $\mathrm{LiH}$ particle suspended at the center of the minimum-B magnetic field is subjected to the effects of a relatively rapidly rising $B$ field. However, calculations show that for the conditions of the experiments the particle should not be affected and no perceptible perturbation of the suspended particle has been observed in tests up to $8 \mathrm{kG}$ center field. When the magnet current reaches a preset increment (50-100 amp) below the steady-state value, the magnet control system delivers a control pulse which arms the laser trigger timer. Then, using the "flicker" technique for detecting when the particle is in the laser focal spot (described in Ref. 17), the laser is triggered when the particle next enters the focal spot. This system has operated with no difficulty in the initial series of tests in which plasmas of $500 \mathrm{eV}$ average energy were produced from particles of $\leq 40 \mu \mathrm{m}$ diameter in magnetic fields of center intensity up to $7.5 \mathrm{kG}$.

\section{Large Particle Suspension}

During this test series, difficulties were encountered in suspending particles larger than $40 \mu \mathrm{m}$, although larger particles had been routinely captured in earlier tests of the suspension system alone. Subsequent investigations have indicated that the difficulty was the result of several interrelated phenomena, all directly or indirectly related to the use of a simple, a.c. heated, bare filament to apply the electrostatic charge to the suspended particle. Due to the very strong dependence of electron emission on temperature, the $60 \mathrm{~Hz} a . c$. heated filament emits bursts of electrons at $120 \mathrm{~Hz}$, and the effect of these bursts on the suspended particle is to induce large oscillations which tend to throw the particle out of the region within which the particles can be stably suspended. When the internal structure of the experimental chamber was at room temperature, this was not necessarily an intolerable effect; however, when the magnet was chilled, the filament, which is positioned near the inner edge of the magnet shell, is subjected to very much greater cooling. This results in much larger temperature variations over one cycle of heating current and, consequently, much more pronounced bursts of emitted electrons. Attempts to override this problem by simply increasing the peak filament temperature were defeated by changes in the potential of the suspension plate nearest to the filament induced by excessive current drain from the high-impedance suspension plate voltage source. A workable solution which has been successfully tested is to use d.c. heating of the filament and to replace the bare filament with a simple, shielded electron gun, with the shield potential allowed to float with respect to ground. With these changes and some minor modifications in the suspension voltage source, the largest particles yet introduced ( $~ 90 \mu \mathrm{m}$ diameter) have been suspended with the system at room temperature, and tests at cryogenic temperatures will be conducted in the near future. 
Under the current experimental conditions, these larger particles are expected to yield somewhat more energetic plasmas (Ref. 18) but an even more important consideration is the production of a greater quantity of plasma (a fully ionized, 40 micron diameter, LiH particle results in $2.5 \times 10^{16}$ ions.) Such plasma quantities are desirable for investigations of the filling of the containment volume of the new magnetic field (see following section) and for investigations to determine the filling requirements of larger devices such as Baseball-II where the volume of the magnetic containment region may be 100 times larger. Continuing experimental investigations on suspending larger particles are currently directed toward more efficient charging of the particles in order to attain a charge to mass ratio sufficient to control the particle during its initial injection.

\section{Plasma Capture and Confinement}

Initial experiments to examine the capture and confinement of laser-produced plasmas within the minimum-B mirror magnetic fields of the new baseball coil magnet were conducted at field strengths ranging from $5.5 \mathrm{kG}$ to $7.5 \mathrm{kG}$. Plasmas were produced by irradiating electrically suspended, 40 micron diameter, LiH particles with 5 nsec, 6 joule pulses from a Nd-glass laser. Integrated charge collectors, time-of-flight probes, and a microwave interferometer were used for diagnostics of the plasmas produced. With no magnetic field, a series of observations of the plasma expansion velocity and quantity of plasma and of the spatial symmetry of these quantities established the correct positioning of the particle in the focused laser beam for the production of high energy, symmetrically expanding plasmas. Plasma energies of 200-500 eV were attained, with particle positioning being the most critical parameter affecting the plasma energy. With the magnetic field on, the observed expansion velocity along the field axis was used to calculate the plasma energy as in Ref. 19 and the microwave interferometer was used to follow the density decay. The observed density decay appeared qualitatively similar to that obtained previously with the $10 \mathrm{~cm}$, cubical array, six coil magnet system, although the microwave signal was cut off for a shorter duration.

This shorter cut-off time in the new field geometry occurs even when the plasma energy and magnetic field strength are the same as in the earlier field geometry so that the $\beta=1$ capture volume should be identical in the two cases. It is of interest to establish whether the shorter cut-off time in the new baseball field was the result of the confined plasma filling the larger magnetic field volume. At present the nature of the transition from $\beta \sim 1$ capture to $\beta<I$ confinement is not well understood so that the extrapolation of laser-produced plasma techniques to filling large experimental devices such as Baseball-II is uncertain. Future experimental observations are expected to clarify this transition process.

With the aid of a fractional fringe shift calibration added to the mic rowave interferometer via a mechanical phase modulator in the reference arm of the interferometer (Ref. 17), phase shifts down to a few hundredths of a radian, corresponding to density-length products approaching $10^{11}$ electrons $/ \mathrm{cm}^{2}$, were measured. For the plasmas generated in the current experiments this permitted the density decay to be 
followed for more than 500 microseconds. The density decay of the confined plasma is observed to be relatively gradual for the first few hundred microseconds and is roughly consistent with Coulomb scattering into the magnetic field mirror loss cones. Following this gradual decay, however, the plasma density decreases sharply, and the plasma disappears precipitously at a time of $\sim 500$ microseconds. This, however, is not an unexpected result, since the ambient gas pressure for these experiments was relatively high $\left(5 \times 10^{-6}\right.$ torr $)$ and calculations of the plasma decay including the effects of charge exchange show that charge exchange collisions will dominate the plasma loss rate when the ratio of the cold neutrals to hot ions in the plasma is equal to the ratio of the scattering cross-section to the charge exchange crosssection $\left(\sim 10^{-3}\right)$. When charge exchange dominates, the plasma will experience "wipe out" as plasma density decays exponentially rather than inversely with time. The high background pressure in these experiments was the result of small leaks in a flexible bellows of the focusing lens mount. This bellows has now been replaced and future experiments at lower ambient pressures ( $\sim 2 \times 10^{-7}$ torr) are expected to show "wipe out" delayed to lower plasma densities later in the plasma decay. Observation of this effect will both establish the mechanism of the enhanced latetime decay and also serve as a check on the burn out calculations for the target plasma investigations.

\section{Neutral Beam Line}

The long range objective of the UARL Laser Initiated Target Experiment (LITE) is to demonstrate the feasibility of creating a hot, dense, mirror confined plasma sustained by energetic neutral injection. While this objective is common to all major mirror experiments, the use of a laser-produced plasma as the target provides an initially dense, highly localized target plasma unencumbered by background neutrals, and the Fokker-Planck calculations of the plasma development indicate that with such a target the feasibility of neutral beam injection can be investigated in an experiment the size of the UARL facility using existing neutral beam technology. Accordingly, a detailed review of the current technology in energetic neutral beam production developed in the programs at ORNL, LLL, and Berkeley has been made and a design effort initiated to achieve a beam for the LITE injection experiments.

A recent AEC Mirror Review Panel has established as near term, interim goals for a neutral injection feasibility experiment the attainment of a neutral injection sustained, steady-state, mirror confined plasma for which $\mathrm{n} \tau \geq 10^{11} \mathrm{~cm}^{-3} \mathrm{sec}$, $T \geq 10 \mathrm{keV}$, plasma lifetime $\geq 10 \mathrm{msec}$, and $\varepsilon=\left(\omega_{p i} / \omega_{j i}\right)^{2} \geq 10 \overline{0}$. On the basis of the

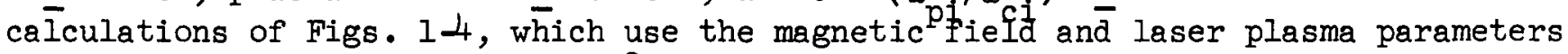
of the UARL experiment, a $5 \mathrm{~mA} / \mathrm{cm}^{2}$ equivalent beam of $10 \mathrm{keV}$ neutrals injected over a cross-sectional area of $2 \mathrm{~cm} \times 10 \mathrm{~cm}$ (to match the projected area of the initially confined laser plasma) will sustain in a background vacuum of $<3 \times 10^{-9}$ torr a steady-state plasma with $\mathrm{n}_{\mathrm{T}} \sim 10^{11} \mathrm{~cm}^{-3} \mathrm{sec}, \mathrm{T}=10 \mathrm{keV} ; \tau=I \mathrm{sec}$, and $\epsilon=200$. These parameters equal or exceed the goal values established by the Review Board and are achieved with a total beam current of 100-200 mA equivalent. State-of-the-art neutral beam technology lies in the tens of amperes range, and the relatively modest 
beam requirements of the UARL LITE program permit the beam line for this effort to be optimized for beam intensity and purity.

The generation of an intense, energetic neutral beam is accomplished by the extraction of ions from a plasma source, electrostatic acceleration of the ions to the desired energy, focusing of the ion beam for maximum intensity at the target area, followed by neutralization of the ions by molecular break-up and charge exchange process in a gas vapor. The resulting focused high energy neutral beam is injected across the magnetic containment field and into the confined plasma. The design of the beam line is thus concerned with the optimization of the ion source, beam extraction and focusing, and beam neutralization which are considered individually in the following sections. In each area the design goal is to achieve the maximum beam intensity consistent with a background gas streaming rate sufficiently small to assure the required vacuum of $\sim 3 \times 10^{-9}$ torr in the target plasma experimental chamber.

\section{Ion Source}

For extraction of an intense ion beam, the ion source should possess a large, uniform density surface capable of high emissive density. Sources of the duoplasmatron type are most commonly used for steady state operation and are the most highly developed. In a duoplasmatron source, the plasma from which the ions are extracted is created by discharge ionization of a gas flow typically at a pressure of $10^{-3}$ torr giving a plasma source density of $\sim 3 \times 1013 / \mathrm{cm}^{3}$. The surface emissive density of such a plazsma as optained from the relation, $j_{e}=n_{i} v_{i}$, where $n_{i}$ is on the order of $\tilde{\mathrm{kT}}_{\mathrm{e}} \mathrm{x} 10^{\frac{1}{2}}$ ions $/ \mathrm{cm}^{2}$ is the ion density in the quasi-neutral bulk plasma and $\mathrm{v}_{i}=$ $\left(\frac{\mathrm{kT}_{\mathrm{e}}}{\mathrm{m}_{\mathrm{i}}}\right)^{\frac{1}{2}}$ is the ambipolar velocity of the ions which cross the sheath to reach the plasma surface. Consequently, the current that can be extracted from these high density sources is generally limited by space charge effects and is given by the Childs-Langmuir law

$$
j=3.85 \times 10^{-8} \frac{\phi^{3 / 2}}{l^{2}} \mathrm{amp} / \mathrm{cm}^{2}
$$

where $\phi$ is the extraction voltage in volts applied across the effective electrode gap spacing in $\mathrm{cm}$. The geometry of the equipotential surface of the plasma from which ions are extracted is determined by the balance of the thermal flux, nv $/ 4$, arriving at the surface with the flux extracted by the applied voltage. For Iow voltage extraction from a high density source, the beam current is limited to that given by Childs-Langmuir law and the larger thermal flux results in a convex plasma surface so that the extracted beam tends to diverge. For high voltage extraction from a low density source, the extracted current is limited by the available thermal flux resulting in a concave plasma surface and a converging, i.e., focused, extracted beam. To extract a well collimated beam of maximum intensity, the plasma density in the source must be adjusted so that the emitting surface is essentially planar or slightly concave. In a high density ion source this can be accomplished by the use of an expansion cup into which the plasma spreads reducing the plasma density to the 
desired value in a controlled manner over a large extraction area.

Most of the development work on ion sources of the duoplasmatron type have been carried out by the beam groups at the Oak Ridge National Laboratory and the Lawrence Livermore Laboratory. At this point, the Duo-PIG-atron source that has been extensively developed at ORNL (Ref. 20) appears the most suitalbe for our requirements, although the MATS-III source currently being tested at LLI may prove equally applicable. The Duo-PIG-atron source consists of a duoplasmatron feeding a PIG discharge, and provides a relatively uniform density plasma over a $5 \mathrm{~cm}$ diameter circular region from which ions can be extracted. The current density attainable at $20 \mathrm{keV}$ across an $0.5 \mathrm{~cm}$ gap is $0.4 \mathrm{amps} / \mathrm{cm}^{2}$ of aperture opening. To extract $100 \mathrm{~mA}$ required for the experiment, requires only a fraction of the available plasma surface, providing great latitude for focusing and gas flux optimization.

\section{Beam Extraction Optics}

The intensity of a beam is inversely related to the product of the beam line length and the angular beam divergence. In view of the beam line length required for vacuum isolation of the experimental chamber from the gas load of the ion source and neutralizer cell, the beam divergence must be small to obtain a high beam intensity at the target plasma. Beam divergence arises principally from space charge blowup, thermal spreading, and lens effects associated with the extraction electrodes. From experimental studies, it has been established that the extracted ion beam is fully space charge neutralized if the background pressure is maintained above $10^{-4}$ torr, and space charge blowup can be effectively eliminated. However, this high background pressure imposes severe constraints on the vacuum system design, requiring several stages of differential pumping between the source and experimental chamber. Thermal spreading of the beam is an inherent residual effect, and the associated angular beam divergence is on the order of the square root of the ratio of the ion temperature in the source to the beam energy. For the energetic beams of interest, the thermal spreading beam divergence is $\leq 10^{-2}-10^{-3}$ radian and is relatively insignificant. The potentially most serious source of beam divergence arises from the lens effect of the extraction optics and must be minimized by proper design of the extraction electrodes.

The lens effect arises in part, from the curvature of the plasma emitting surface as discussed in the previous section, and in part, from field line leakage associated with the aperture openings. In general, for a lens system that is symmetric about an axis, the potential near the axis may be represented by an even power series

$$
\phi(r, z)=\psi(z)-\frac{1}{2} \psi^{\prime \prime}(z) r^{2}+\frac{1}{24} \psi^{I V}(z) r^{4}-\ldots
$$

where $\psi$ is the potential on the axis $(r=0)$ and the superscript denotes derivative with respect to the axial coordinate $z$. For a cylindrically symmetric system, i.e., a circular aperture, the potential distribution will be cylindrically symmetric, the resulting beam divergence is radial, and the beam intensity at the target will 
decrease inversely as the square of the beam line length-beam divergence product. Equation 31 for the extraction potential, however, also applies to a two-dimensional system with reflective symmetry about the axis, i.e., an infinite slit aperture (or realistically, a slit whose length is much larger than its width). In such a system the potential distribution is. independent of position along the length of the slit and is more appropriately described in Cartesian coordinates with the $r$ coordinate in the direction of the slit width. The beam divergence of this system is one-dimensional, and the beam intensity varies inversely as the first power of the beam line length-beam divergence product. Thus, from lens effect considerations alone, slit apertures are potentially capable of delivering a higher intensity beam than circular apertures. Slit apertures possess the additional advantages of higher transparency as well as greater amenability to designs with internal water cooling for high power operations, and are incorporated in the present beam line design.

As a basis for the development of a slit extraction system, analytical calculations have been made of the beam divergence associated. with slit aperture as a function of the slit width and electrode spacing. The orbit equation for the extracted ions is obtained by substitution of the potential distribution of Eq. 31 into the equation of motion

$$
M \frac{d^{2} r}{d t^{2}}=z e \nabla \phi
$$

for ions of mass $M$ and charge Ze. Making use of energy conservation

$$
\frac{M}{2}\left[\left(\frac{d x}{d t}\right)^{2}+\left(\frac{d z}{d t}\right)^{2}\right]=z_{e} \phi
$$

the orbit equation to lowest order is

$$
\frac{d^{2} x}{d z^{2}}+\frac{\psi^{\prime}}{2 \psi} \frac{d x}{d z}+\frac{\psi^{\prime \prime}}{2 \psi} x=0
$$

which may be integrated approximately to yield the simple result

$$
\frac{d x}{d z}=\frac{1}{4} \frac{w}{l}=\frac{A}{4}
$$

where $A$ is the aspect ratio of the slit extraction system defined as the ratio of the width of the slit $w$ to the interelectrode spacing $l$. The beam divergence associated with the slit aperture is given by

$$
\alpha_{l}=\tan ^{-1} \frac{d x}{d z} \sim \frac{d x}{d z}=\frac{A}{4}
$$

and small beam divergence requires a small aspect ratio. Since the area of the aperture through which the beam is extracted is proportional to the slit aspect ratio, the beam divergence and beam current requirements impose conflicting demands on the slit design. The optimum slit extraction electrode configuration is obtained when the lens effects of the optics are comparable with the beam divergence from other sources, principally thermal spreading. The thermal spreading beam divergence is 
given to a good approximation by the square root of the ratio of the ion thermal energy $\mathrm{E}$ to the extraction energy, $\mathrm{Z} \varphi$

$$
a_{\dagger} \sim\left(\frac{E}{z \phi}\right)^{1 / 2}
$$

Since there is little point in minimizing the beam divergence associated with the lens effect beyond the inherent residual beam divergence associated with the thermal spreading, the optimum width for a slit system is obtained by equating the two beam divergences to yield

$$
w_{\text {optimum }} \sim 4\left(\frac{E}{z \phi}\right)^{1 / 2} \ell
$$

Using this result and Eq. 30, current per unit length that can be extracted from such an optimum slit is

$$
\begin{aligned}
I_{\text {slit }} & =3.85 \times 10^{-8} \frac{\phi^{3 / 2}}{\ell^{2}} w_{\text {opt }} \\
& =1.54 \times 10^{-7} \frac{\phi E^{1 / 2}}{z^{1 / 2} \ell} \text { (amp) }
\end{aligned}
$$

The slit extraction system required to form a $10 \mathrm{keV}$ beam of $\mathrm{H}^{\circ}$ by the extraction of $20 \mathrm{keV} \mathrm{H}_{2}{ }^{+}$may be calculated from these results as an example pertinent to the LITE program. The thermal energy of the beam ions may be taken as $\sim 10 \mathrm{eV}$ arising from the dissociation energy of $\mathrm{H}_{2}{ }^{+}$and from $\mathrm{Eq} .38$ the optimum slit width is w $\sim 0.1 l$. Taking the interelectrode spacing $\ell$ as $0.5 \mathrm{~cm}$, the beam current that can be extracted from the aperture is $\sim 20 \mathrm{~mA}$ per $\mathrm{cm}$ of slit length. As discussed in Section, a total slit length of 10-20 cm would be required for a beam current of 100-200 mA into the plasma and would be accomplished by the use of extraction electrodes consisting of an array of multiple parallel slits. The operation of such slit arrays has been investigated experimentally using a reflex arc plasma source and found to be in good agreement with the beam current and divergence results of the analysis.

\section{Neutralizer Cell}

Neutralization of the ion beam is accomplished by passing the extracted beam of hydrogen ions (predominantly $\mathrm{H}^{+}$and $\mathrm{H}_{2}^{+}$) through a gas cell where collisional molecular breakup and charge transfer processes convert the ions to energetic neutrals. Since the processes involved have cross-sections of $\sim 10^{-15} \mathrm{~cm}^{2}$ effective neutralization requires a mean free path, $\lambda=(n \sigma)^{-1}$, through the neutralizer cell of unity, i.e., a vapor line density of $\geq 10^{15} \mathrm{~cm}^{-3}$. To achieve this line density in a compact system, the neutralizer celi must necessarily operate at a relatively high gas density. The basic concern in the design of the neutralizer is to achieve the required vapor line density while minimizing the density of streaming vapor into the experimental plasma chamber. The streaming density at a distance I from a source of density $n_{0}$ through a source aperture of characteristic dimension $d$ is given by

$$
n=\frac{1}{s}\left(\frac{d}{L}\right)^{2} n_{0}
$$


where $S$ is a numerical constant whose value depends on the velocity distribution of the vapor in the source. For a fully Maxwellian collisional gas cell, the streaming density is determined by the Knudson effusion flux, and $S$ has the minimum value of 32. Optimization of the neutralizer cell requires maximizing the value of $\mathrm{S}$. This can be achieved by making the velocity distribution of the neutralizing gas highly directional and oriented away from the beam direction. To this end, a supersonic jet, giving a highly directional flow with reduced random thermal velocity directed across the beam can be used to provide the neutralizing vapor. This type of neutralizer has been employed with some success (Ref. 21); however, in order to achieve the line density required for neutralization, the jet must be driven with a large pressure gradient which results in large mass flow and considerable spreading of the jet, and the pumping capacity required to maintain a high vacuum in the presence of the large load makes this approach rather unattractive for application to the LITE experiments (Ref. 22).

It should be noted that in order to avoid blow up of the ion beam, the beam must be space charge neutral which requires that the background pressure between the ion source and neutralizer be kept above $10^{-4}$ torr. Accordingly, there is little benefit in minimizing the streaming flux from the vapor jet below the effusion flux from a source with density $3 \times 10^{12} \mathrm{~cm}^{-3}$, corresponding to $10^{-4}$ torr. To achieve the required neutralizing line density of $\geq 10^{15} \mathrm{~cm}^{-2}$ in a reasonably compact system, the vapor density must be comparable with or greater than $10^{14} \mathrm{~cm}^{-3}$. Therefore, the streaming flux can be reduced to that due to background effusion if the value of $S$ for the neutralizer vapor can be increased by an order of magnitude. A technique to achieve this while maintaining a reasonable mass flux is the cross beam neutralizer developed at the Lawrence Livermore Laboratory. In this technique, the velocity vector of the neutralizer vapor is partially collimated by flow through an array of very fine capillaries (Ref. 23). Typically, a vapor density of $10^{14} \mathrm{~cm}^{-3}$ can be achieved at a distance $2.5 \mathrm{~cm}$ away from an array of $0.1 \mathrm{~mm}$ bore capillaries having a transparency of $\sim 50 \%$. By using a readily condensable gas such as water vapor the flux can be effectively pumped by a liquid nitrogen cooled surface, and the streaming density is experimentally about an order of magnitude below that for a Maxwellian gas cell at the same density. Additional refinements are also possible: by tilting the jet towards the upstream direction of the beam, the vapor density downstream is further reduced by a factor of four, and an auxiliary jet of gas which does not charge exchange readily with hydrogen ions can be used to alter the composition of the streaming vapor to reduce its interaction with the target plasma. The combined use of these techniques should permit the streaming vapor flux to be reduced to a tolerable level. 


\section{Beam Line}

The requirements for the beam line output are the delivery at the plasma target of a neutral beam current of $5 \mathrm{~mA} / \mathrm{cm}^{2}$ over an oblong area $\sim 2 \mathrm{~cm} \times 10-20 \mathrm{~cm}$ for a total beam current of 100-200 $\mathrm{mA}$ while maintaining the combined streaming and effusion flux below that corresponding to a background density of $10^{8} \mathrm{~cm}^{-3}$. To meet these requirements the ion source must operate at $\sim 10^{-3}$ torr which imposes important vacuum design constraints on the beam line system. A preliminary beam line design layout developed on the basis of these constraints is shown in Fig. 9 and which consists of five sections: ion source, neutralizer, drift tube; experimental chamber, and beam dump. In this design each section is isolated by gate valves, and the entire beam line is differentially pumped to maintain the required high vacuum in the experimental chamber.

In the design the ion source is located at a distance of $250 \mathrm{~cm}$ from the center of the experimental chamber. Utilizing slit apertures with a beam divergence of $\alpha \sim 3 \times 10^{-2}$ radians, the cross sectional area of the unapertured beam at the target will be about $175 \mathrm{~cm}^{2}$. Since the target plasma is expected to have a projected area of $20-50 \mathrm{~cm}^{2}$, the delivery of $100-200 \mathrm{~mA}$ of beam current into this area $\left(5 \mathrm{~mA} / \mathrm{cm}^{2}\right.$ ) will require the extraction of $\sim 1.0 \mathrm{amp}$ from the source. This is accomplished with $40 \mathrm{~cm}$ total length of $0.06 \mathrm{~cm}$ wide slits arranged in multiple rows with a total transmission area of $2.4 \mathrm{~cm}^{2}$. With the ion source operating at a density of $3 \times 10^{13}$ $\mathrm{cm}^{-3}$, the flux of streaming particles from the $2.4 \mathrm{~cm}^{2}$ aperture reaching the center of the experimental chamber will correspond to a background density of $\sim 1.15 \times 10^{7}$ $\mathrm{cm}^{-3}$ which is well below the required level.

The neutralizer in the design of Fig. 9 is maintained at a background pressure of $10^{-4}$ torr. There are two types of gas load in this region, water vapor from the vapor jet and $\mathrm{H}_{2}$ from the ion source. The water vapor jet has a density of $10^{14} \mathrm{~cm}^{-3}$ over a length of $30 \mathrm{~cm}$ to achieve a line density of $3 \times 10^{15} \mathrm{~cm}^{-2}$. At this line density, the conversion is approximately $90 \%$ of the equilibrium value of each incident $\mathrm{H}_{2}^{+}$converting to $1.6 \mathrm{H}^{\circ}$. This results in a gain of 1.4 particles per incident ion in the neutralization process which should compensate for some particle loss due to scattering. To achleve the desired line density, the capillaries are driven with a throughput of $3 \times 10^{15}$ particles/sec per capillary, and $\sim 10^{6}$ capillaries are employed for a total throughput of $3 \times 10^{21}$ particles/sec corresponding to a mass flow of $\sim 0.1 \mathrm{gm} / \mathrm{sec}$ of water. Since water vapor is readily condensed out at liquid nitrogen temperature, this load is handled by cryogenic pumping on the liquid nitrogen temperature surface that surrounds the vapor cells. Hydrogen gas from the source operating at a density of $3 \times 10^{13} \mathrm{~cm}^{-3}$ comprises an effusion flux of $2.8 \times 10^{18}$ particles/sec through the $2.4 \mathrm{~cm}^{2}$ source aperture. In addition, $90 \%$ of the extracted I A of beam current is apertured out at this point and constitutes an additional gas load of $3 \times 10^{18}$ particles/sec. This gas load must be handled by the diffusion pump, and to maintain the background density at $\leq 3 \times 10^{12} \mathrm{~cm}^{-3}$ (10-4 torr) requires a pumping speed of $\geq 2 \times 10^{3}$ litres/sec. With the background density maintained at $3 \times 10^{12} \mathrm{~cm}^{-3}$, the flux of streaming particles at the center of the experimental chamber from the neutralizer through the $5 \mathrm{~cm}$ aperture opening located at $200 \mathrm{~cm}^{2}$ 
away from the chamber is $3.75 \times 10^{6} \mathrm{~cm}^{-3}$.

The drift tube section serves several functions. It maintains the physical separation between the source and the experimental chamber required to reduce the streaming flux from the neutralizer to the required level. It also sets up a differentially pumped region to drop the pressure from the $10^{-4}$ torr of the neutralizer to $3 \times 10^{-9}$ in the experimental chamber. In addition, the drift tube contains a deflection magnet to remove the unneutralized portion of the beam and prevent it from entering and contaminating the high vacuum experimental chamber. The gas load into the first section of the drift tube consists of the $5 \times 10^{17}$ particles/sec effusion flux from the neutralizer and the $3 \times 10^{17}$ particles/sec unneutralized portion of the beam deflected by the bending magnet. This region is evacuated by a diffusion pump, but the bulk of the pumping speed comes from getter pumping on the $\sim 1.5 \times 10^{3} \mathrm{~cm}^{2}$ surface of the inside wall of the chamber at room temperature and on the back side of the dewar at liquid nitrogen temperature. For hydrogen gas, the sticking probability of a gettering surface is $\sim 0.1$ at room temperature and 0.3 at LN temperature. The pumping speed available in this section is, then, $5 \times 10^{6} \mathrm{~cm}^{3} /$ sec, which will maintain the background density in this region at $10^{11} \mathrm{~cm}^{-3}$ corresponding to a pressure of $\sim 3 \times 10^{-6}$ torr. The liquid nitrogen dewar defines a high vacuum second section of the drift tube section which is further pumped by gettering on the surface of the dewar at liquid nitrogen temperature. The gas load into this region is restricted to the flux that enters through a $10 \mathrm{~cm}^{2}$ beam defining aperture. This gas load is pumped by an area of $\sim 8 \times 10^{3} \mathrm{~cm}^{2}$, and, with a sticking probability of 0.3 , the pressure differential between the two regions, determined by the ratio of the aperture area to the effective pumping area, is a factor of $\sim 250$ and the pressure in the high vacuum region will be dropped to $\sim 10^{-8}$ torr. A final beam 1 defining aperture on the exit side of the drift tube limits the gas load into the experimental chamber to $\sim 10^{14}$ particles/sec, requiring a pumping speed of only $\sim 10$ litres/sec to maintain $3 \times 10^{-9}$ torr in the experimental chamber.

As the beam passes through the target, only about $1 \%$ of the beam will be trapped by charge exchange and ionization collisions, while the remaining $99 \%$ passes on through the experimental chamber. The beam itself, thus, constitutes a gas load of $\sim 6 \times 10^{17}$ particles during the $\sim 1 \mathrm{sec}$ beam pulse. This load is pumped in the beam dump which contains a slanted gettering surface of $\sim 50 \mathrm{~cm}^{2}$ at liquid nitrogen temperature. The surface coverage per pulse is $10^{16}$ particles $/ \mathrm{cm}^{2}$ which is comfortably below the typical saturation value of $10^{17}$ particles $/ \mathrm{cm}^{2}$ for a freshly coated surface.

On the basis of this beam line layout, it appears that $100-200 \mathrm{~mA}$ of neutral beam current can be delivered onto the target without exceeding the background gas density in the experimental chamber required for steady-state operation. This is accomplished within the current state of beam technology by designing each component system to assure minimum gas load contamination. A beam line developed from this layout will permit experimental investigation of the neutral injection approach to establish a fusion condition plasma in a mirror confinement field. 


\section{FUTURE PROGRAM}

Mirror confinement systems comprise one of the four main approaches under investigation for controlled fusion power generation. Each has made significant progress toward this goal, although none at present is understood well enough to offer a direct path to a demonstration of feasibility and a prototype powerplant system. Two questions appear central to the development of open system confinement. What are the stabilizing effects of increased density for a mirror confined plasma and to what limiting $B$ value do these extend? Can interaction of an energetic neutral beam with a dense target plasma balance the loss cone loss to establish a steady state, high density mirror confined plasma? The future LITE program at UARL is directed to address both of these questions examining the decay of a dense, mirror confined, laser produced plasma in a baseball coil minimu-B containment field and using the resulting confined plasma as the target for an energetic neutral injection beam.

Analysis of the magnetic field capture and transition to mirror confinement predicts a maximum temperature above which the plasma is lost by effusion during the $\beta \sim 1$ capture phase and no plasma remains to be mirror confined. For a high- $\beta$ plasma trapped in an open-ended system, the thickness of the sheath determines the size of the aperture through which the plasma can escape at the ends of the magnetic field. The thickness and structure of the plasma boundary sheath has been inferred in experiments on the capture of laser produced plasmas from the diamagnetic flux coil signals and the duration of the high- $\beta$ capture phase. On the basis of calculations of the anisotropic plasma-magnetic field interaction and of the resulting equilibrium captured plasma configuration, flux coil measurements will be made for times well beyond the initial transverse trapping to investigate the length of time the magnetic field remains excluded from the plasma. In addition, the amount of plasma remaining when the plasma becomes mirror confined can be evaluated and compared with the captured plasma decay predicted for different values of the sheath thickness to determine the sheath dimensions which best fit the observed plasma development. Investigations of the plasma lifetime as a function of temperature both above and below the calculated critical temperature ( $1 \mathrm{keV}$ ) will be used to test the model of the plasma capture process. The existence of a maximum temperature for effective mirror contalnment is an inherent part of our present understanding of the capture and confinement of a laser produced plasma in a mirror containment field, and experimental observation of the critical temperature will serve as a direct test of the plasma capture model.

In order to be mirror confined, the initially trapped $\beta \sim 1$ plasma must pass through a transition regime during which the magnetic containment field diffuses into the plasma. Although the plasma is neither trapped in the high-s sense nor yet adiabaticaliy mirror confined, on the basis of the prior mirror confinement results the plasma apparently remains stable during this transition phase. Both 
plasma density and flux coil measurements will be performed on plasmas contained in the larger, baseball coil, minimum-B confinement field to examine the plasma behavior during this regime and to further clarify the plasma-magnetic field mixing process. The results of these studies will form a basis on which the scaling of laser produced plasmas to larger confinement geometries can be made.

The apparent classical mirror confined laser plasma decay observed in the earlier experiments is a most important result and, as it bears directly on the feasibility of mirror reactor systems, will be the subject of a major portion of the future investigations. A primary measure of the stability of the plasma will be a comparison of the measured plasma density decay with the predictions of the FokkerPlanck calculations. With the increased field magnitude and length of the baseball coil system and consequent higher non-adiabaticity limit ( $12 \mathrm{keV}$ ), these measurements can now be extended up to the critical capture temperature of $\sim \mathrm{IkV}$ to examine the temperature dependence of the plasma decay over a wider temperature range and to permit measurements on longer lived, more slowly decaying plasmas. In addition, qualitative information on the plasma stability may be obtained by monitoring the electromagnetic radiation emitted from the plasma.

The long range objective of the program continues to be the demonstration of the feasibility of sustaining a fusion condition plasma in a minimum-B mirror magnetic field by the injection of energetic neutrals. The high density attainable with the laser produced target plasma permits the investigation of the upper branch $S$ curve regime in which a mirror reactor must operate. Survey calculations will be extended to include a wider range of plasma and beam parameters to define optimum operating conditions. To facilitate the experimental investigation, detailed designs and development of the beam line components will be carried out in cooperation with and utilizing the experience and technology at the national laboratories. The beam line development will be conducted on a separate installation and upon completion of the plasma capture and decay investigation will be coupled to the confinement chamber for the neutral injection heating studies and the experiments to achieve a hot, dense, steady state, mirror confined plasma sustained by neutral injection. 


\section{REFERENCES}

1. Haught, A. F., D. H. Polk, J. C. Woo, W. J. Fader, R. G. Tomlinson, and R. A. Haas: Production of Plasmas for Thermonuclear Research by Laser Beam Irradiation of Solid Particles, Semiannual Report, July, 1972, UARL L920365-17, COO-3547-2, Prepared under Contract AT(11-1)-3547. for the U. S. Atomic Energy Commission. July, 1972.

2. Killeen, J. and A. H. Futch: J. Comp. Phys., 2, 236 (1968).

3. Killeen, J. and K. D. Marx: Methods of Comp. Phys., Vol. 9, 421, Academic Press, N. Y. (1969).

4. Fite, W. L., A. C. H. Smith and R. F. Stebbins, Proc. Roy. Soc., A 268, 527 (1962).

5. Simon, A., Phys. Fluids 2 , 336 (1959).

6. Haught, A. F., D. H. Polk, J. C. Woo and W. J. Fader: Production of Plasmas for Thermonuclear Research by Laser Beam Irradiation of Solid Particles, Semiannual Report, Jan. 1971. UARL K920365-14, NYO-3578-14, prepared under Contract AT(30-1)-3578 for the U. S. Atomic Energy Commission, January, 1971.

7. Tang, W. M., L. D. Pearlstein and H. L. Berk, Phys. of Fluids, 15, 1153 (1972)。

8. Post, R. F. and M. N. Rosenbluth, Phys. of Fluids, 9, 730 (1966).

9. Berk, H. L., L. D. Pearlstein and J. G. Cordey, Phys. of Fluids, 15, 891 (1972).

10. Dory, R. A., G. E. Guest and E. G. Harris, Phys. Rev. Letters, 14, 131 (1965).

11. Gerardo, J. B., J. T. Verdeyen and M. A. Gusinow, J. Appl. Phys., 36 (1969).

12. Herold, M. and F. C. Jahoda, Rev. Sci. Instr., 40, 145 (1969).

13. Collins, S. A. Appl. Opt., 3, 1263 (1964).

14. Baldwin, D. E. and J. D. Callen, Phys. Rev. Letters 28, 1686 (1972).

15. Rosenbluth, M. N. and R. F. Post, Phys. of Fluids $\underline{8}, 547$ (1965).

16. Damm, C.C., J. H. Foote, A. H. Futch, Jr., A. L. Gardner, F. J. Gordon, A. I. Hunt, and R. F. Post, Phys. of Fluids 1 , 1472 (1965). 


\section{REFERENCES (cont'd)}

17. Haught, A. F., D. H. Polk, J. C. Woo, W. J. Fader, R. G. Tomlinson, and R. A. Haas: Production of Plasmas for Thermonuclear Research by Laser Beam Irradiation of Solid Particles, Semiannual Report, January, 1972, UARL L920365-16, C00-3547-1, Prepared under Contract AT(11-1)-3547 for the U. S. Atomic Energy Commission, January, 1972 .

18. Haught, A. F., D. H. Polk, J.C. Woo and W. J. Fader: Production of Plasmas for Thermonuclear Research by Laser Beam Irradiation of Solid Particles, Semiannual Report, July, 1969, UARL H920365-11, NYO-3578-11, Prepared under Contract AT(30-1)-3578 for the U. S. Atomic Energy Commission, July, 1969.

19. Haught, A. F., D. H. Polk, and W. J. Fader: Production of Plasmas for Thermonuclear Research by Laser Beam Irradiation of Solid Particles, Semiannual Report, June, 1967, UARL F920365-6, NYO-3578-6, Prepared under Contract AT(30-1)-3578 for the U. S. Atomic Energy Commission, June, 1967.

20. Davis, R. C., O. B. Mogan, L. D. Stewart, W. L. Stirling and H. K. Forsen, ORNL-TM-3472 (JuIy 1971).

21. Borovik, E. S., F. I. Busol, V. B. Yuferov and E. I. Skibenko, Sov. Phys. Tech. Phys. 8, 724 (1964), also: Butusov, V. I., P. A. Mukhin and V. S. Svishchev, Sov. Phys. Tech. Phys. 12, 1331 (1968).

22. Myall, John 0., UCRL-51089 (July 6, 1971).

23. Goodman, R. K. and A. Hunt, Rev. Sci. Instr. 42, 1359 (1971). 


\section{LIST OF FIGURES}

Figure 1 Neutral Injection Laser-Produced Target Plasma

Figure 2 Target Plasma Distribution Functions

Figure 3 Neutral Background Burnout

Figure 4 Plasma Density vs. Beam Current

Figure 5 Critical Radius

Figure 6 Transmission Ring Resonator Laser Diagnostic

Figure 7 Experimental Four Mirror Ring Resonator

Figure 8 R.F. Antenna

Figure 9 Magnet Power Supply and Control System

Figure 10 Energetic Neutral Beam Line for Laser Initiated Target Experiment 
NEUTRAL INJECTION LASER - PRODUCED TARGET PLASMA

INITIAL CONDITIONS: $n(0)=5 \times 10^{15} \mathrm{kT}(0)=500 \mathrm{eV} \mathrm{R}=2$

$I=0.1$ AMP EQUIV.
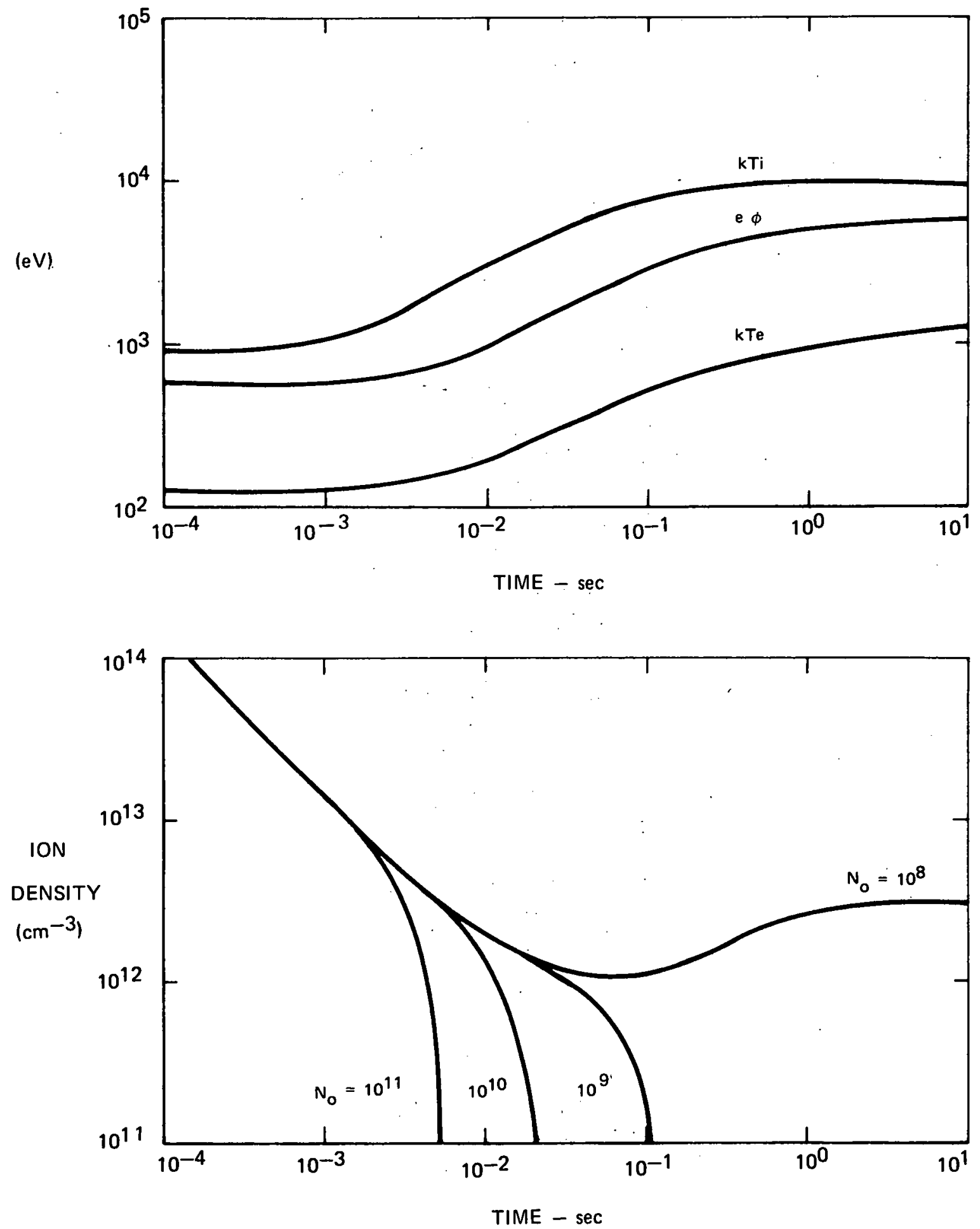

FIG. 1 


\section{TARGET PLASMA DISTRIBUTION FUNCTIONS}

INITIAL CONDITIONS: $n(0)=5 \times 10^{15} \mathrm{~cm}^{-3} \mathrm{kT}(0)=500 \mathrm{eV} \mathrm{R}=2$

$I=0.1 A \quad E_{B}=10 \mathrm{keV} \Delta E_{B}=2 \mathrm{keV}$



\section{NEUTRAL BACKGROUND BURNOUT}

$N_{O}=10^{8} \mathrm{~cm}^{-3}$
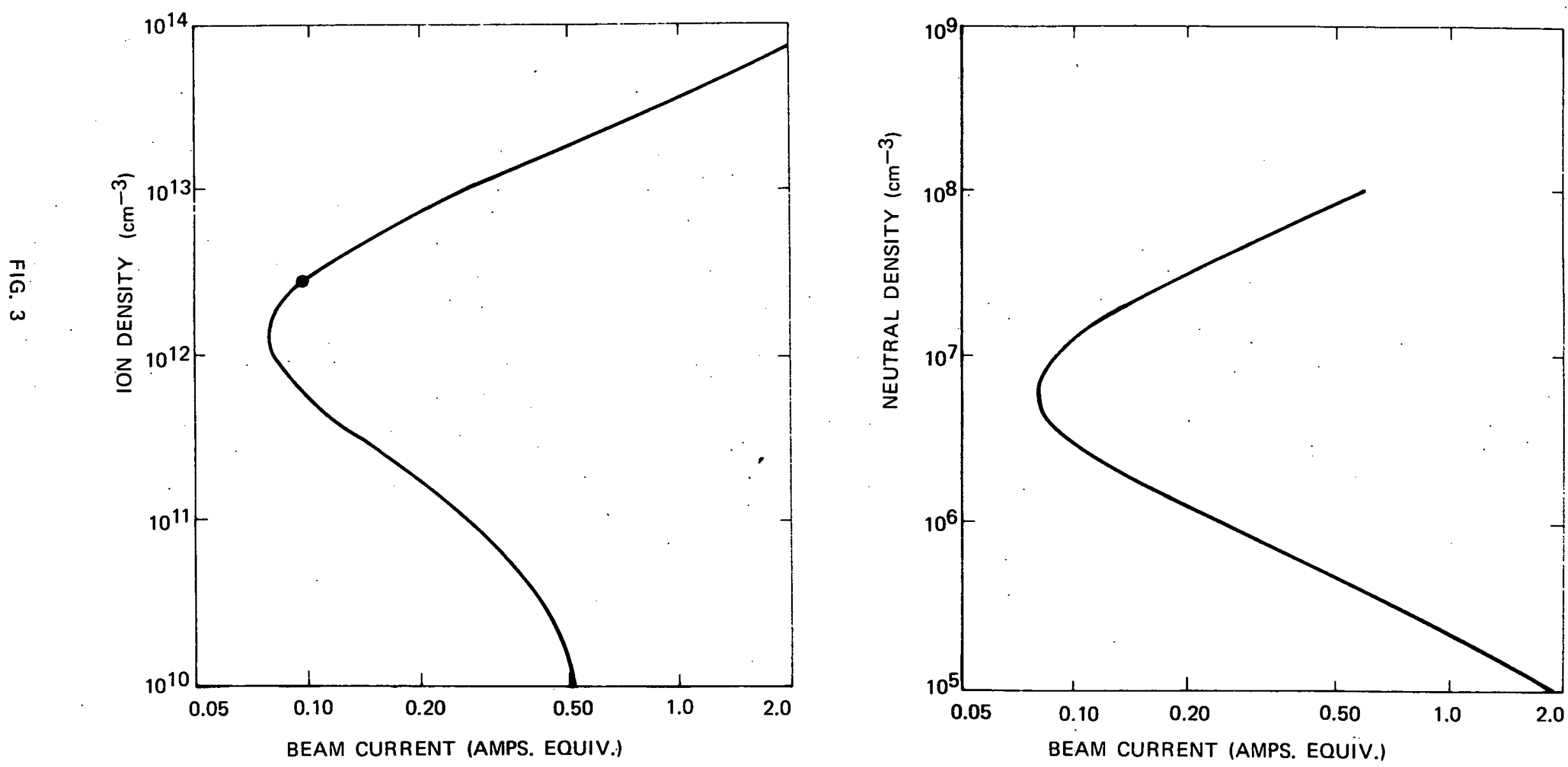
PLASMA DENSITY VS. BEAM CURRENT

())

$T=10 \mathrm{KeV}$

LENGTH $=20 \mathrm{~cm}$

RADIUS $=2 \mathrm{~cm}$

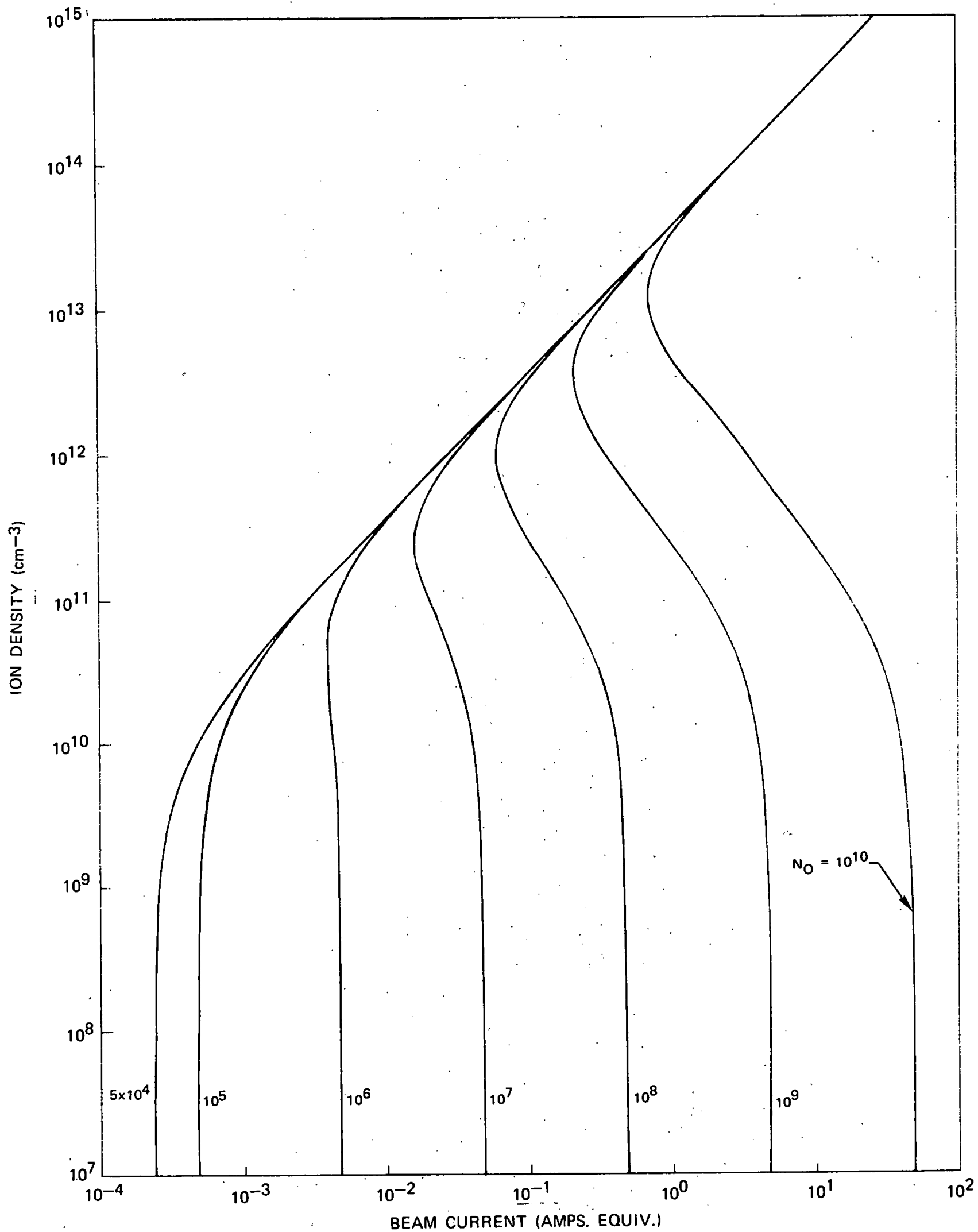

FIG. 4 
CRITICAL RADIUS

MIRROR RATIOO $=\mathbf{2}$

$\mathrm{Ti} / \mathrm{Te}=\mathbf{5 . 0}$

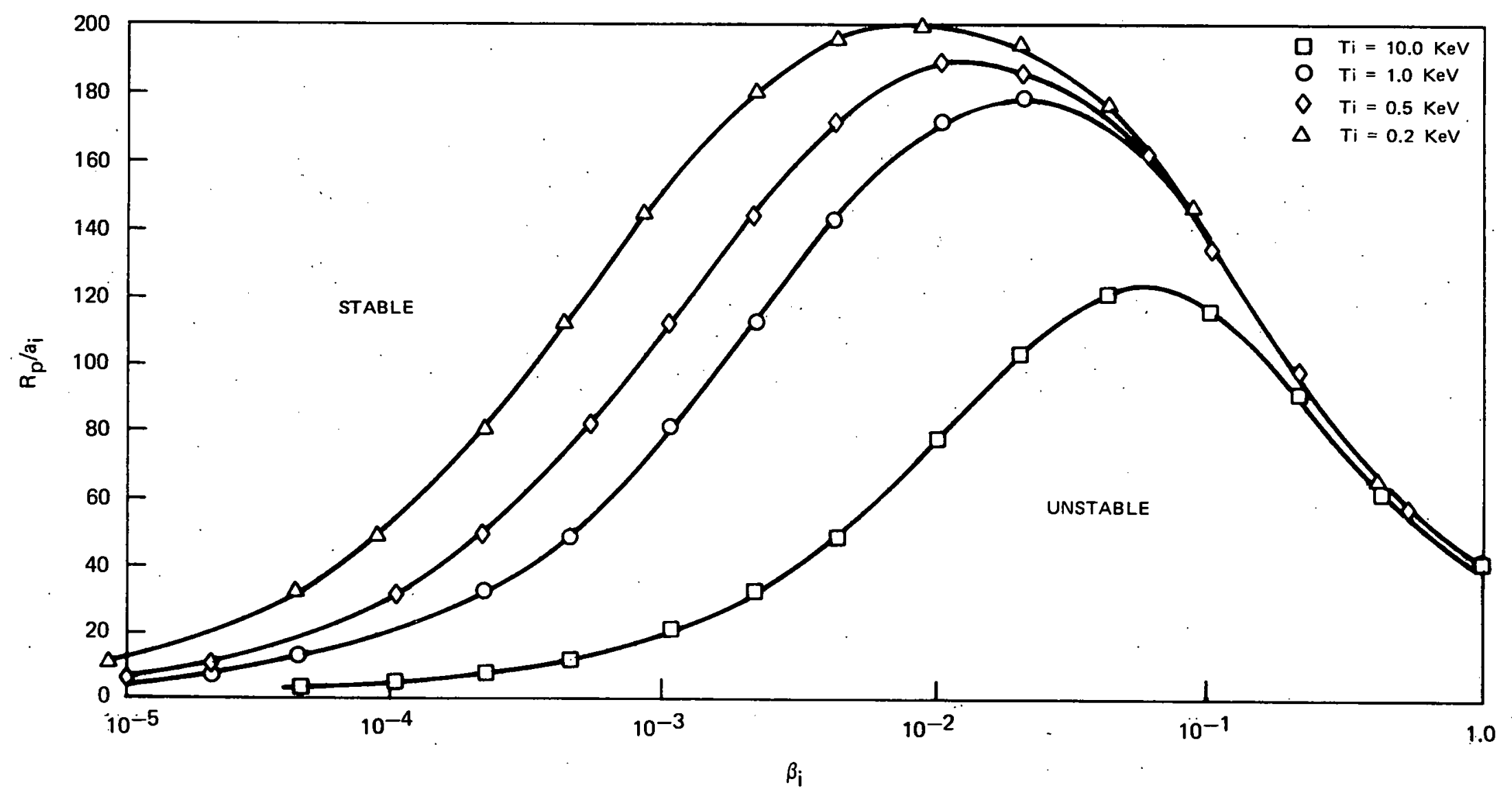




\section{TRANSMISSION RING RESONATOR LASER DIAGNOSTIC}

$10.6 \mu$ OSCILLATOR

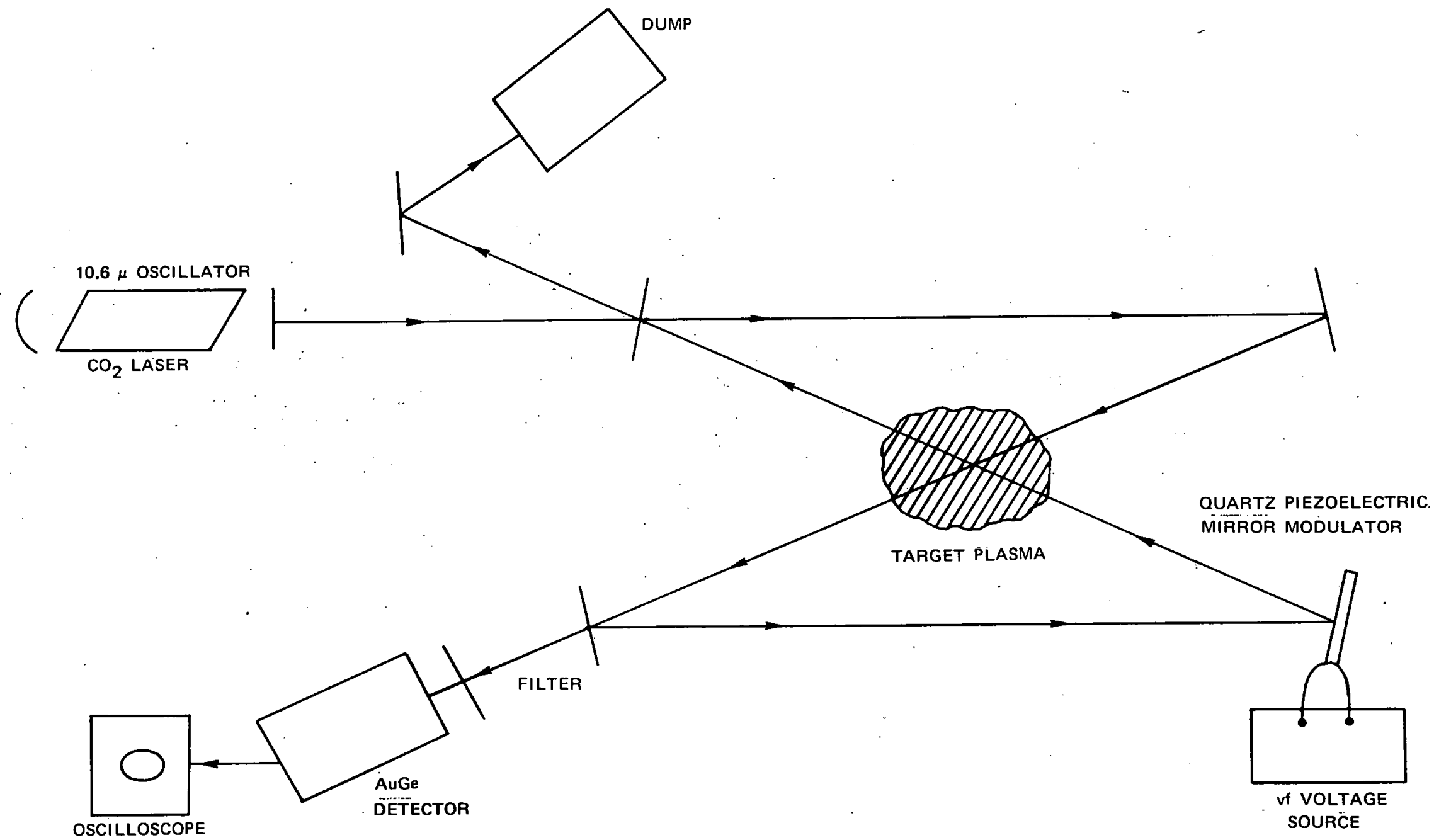




\section{RF ANTENNA}

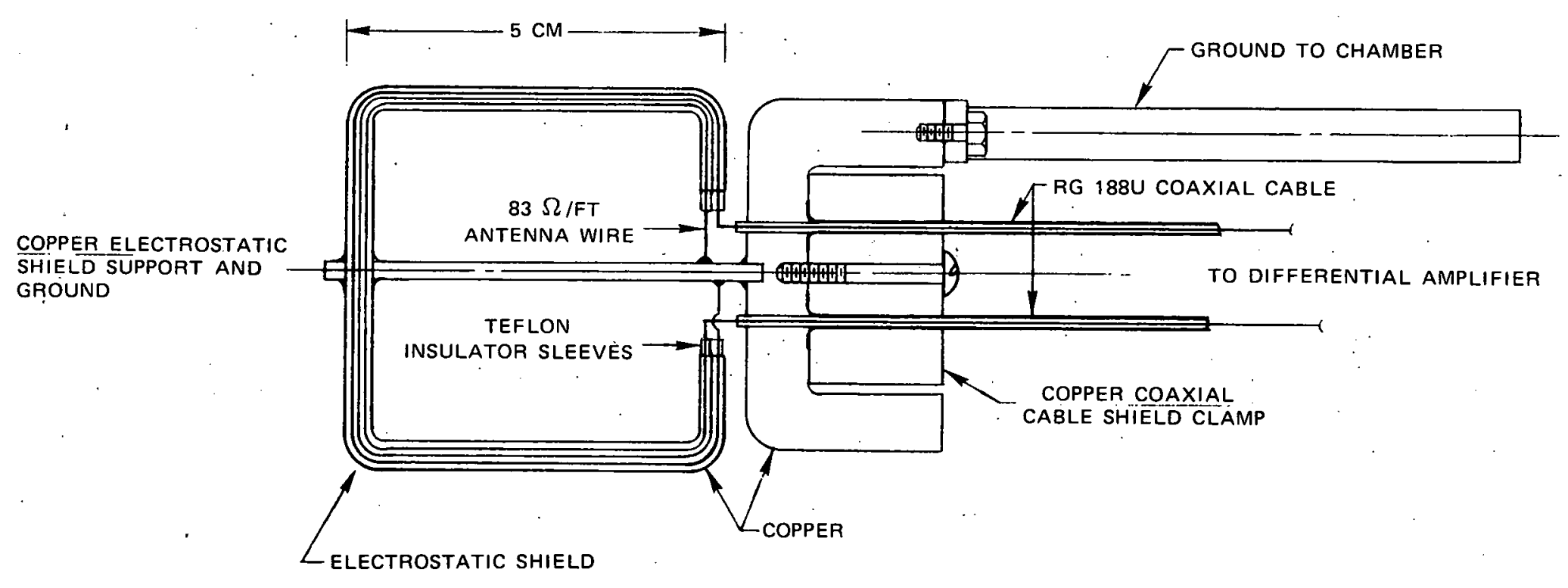

$50 \Omega$ NOMINAL INPUT IMPEDANCE EACH LOOP 
A. CIRCUIT

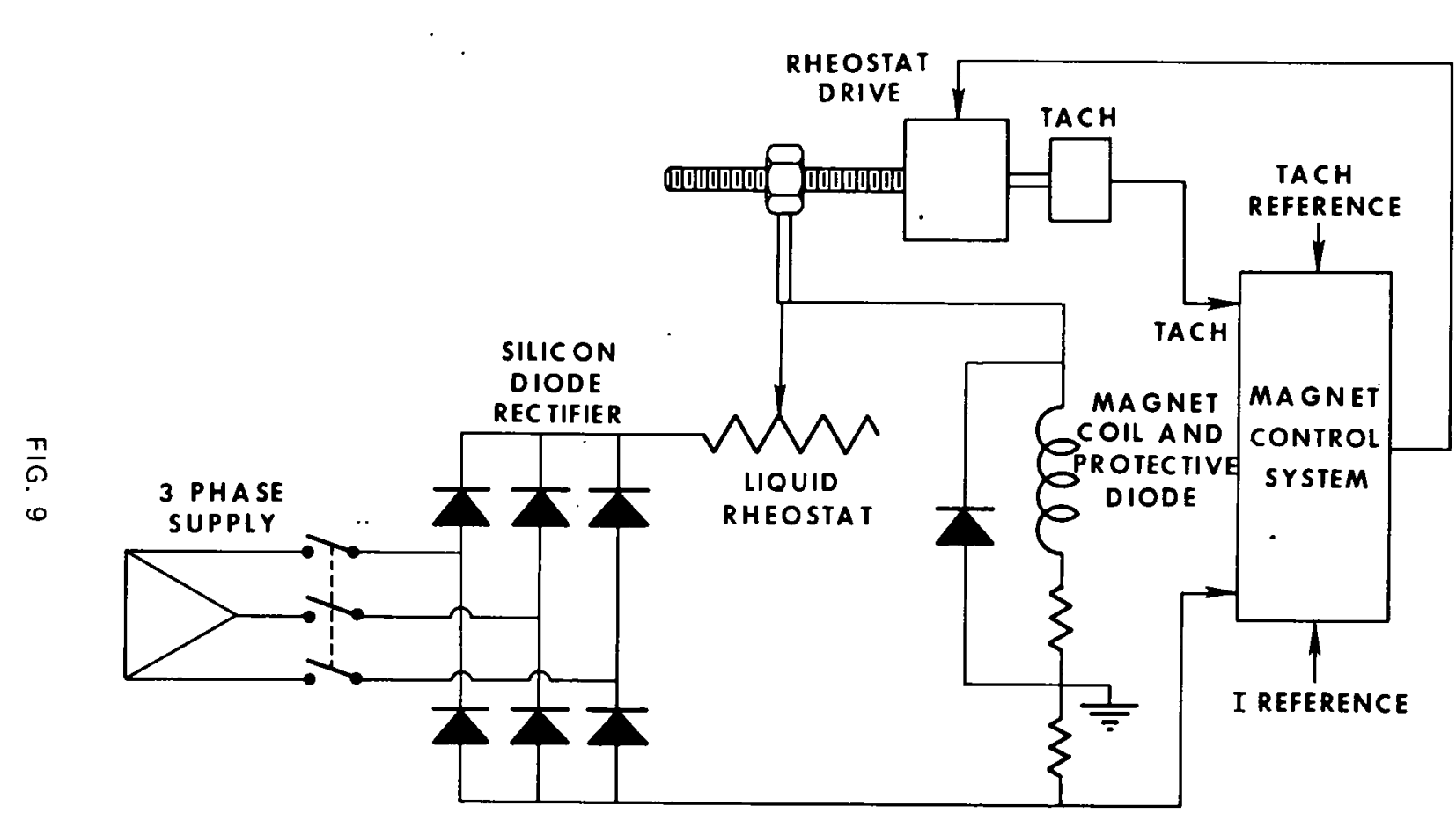

\section{B. MAGNET CURRENT}

United Aircraft
Research Laboratories 
IENERGETIC NEUTRALB BAM LINE FOR LASER INITIATED TARG ETEXPERIMENT

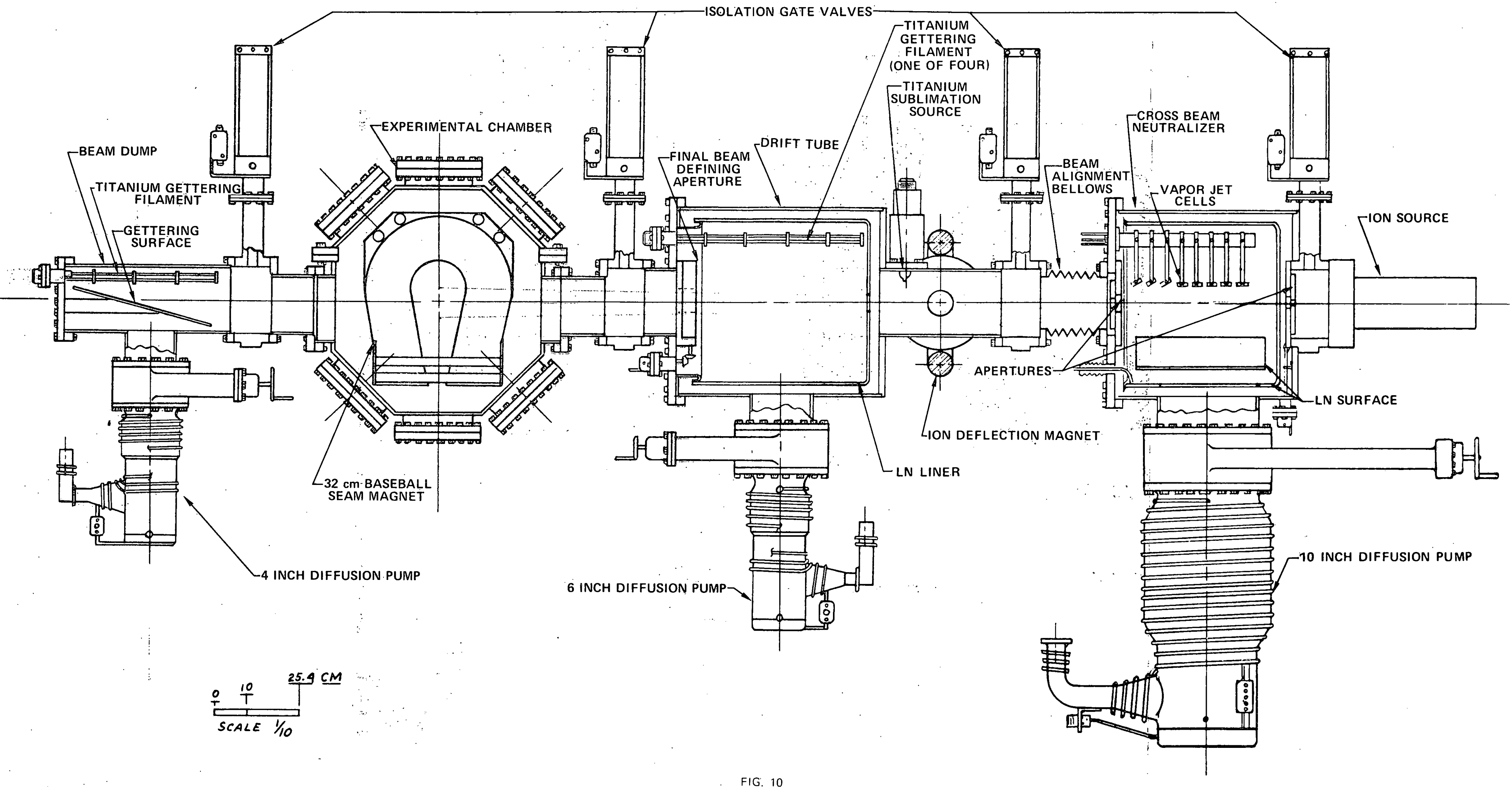

